TRANSACTIONS OF THE

AMERICAN MATHEMATICAL SOCIETY

Volume 364, Number 11, November 2012, Pages 6071-6108

S 0002-9947(2012)05609-1

Article electronically published on April 30, 2012

\title{
A T(1)-THEOREM IN RELATION TO A SEMIGROUP OF OPERATORS AND APPLICATIONS TO NEW PARAPRODUCTS
}

\author{
FRÉDÉRIC BERNICOT
}

\begin{abstract}
In this work, we are interested in developing new directions of the famous $T(1)$-theorem. More precisely, we develop a general framework where we look to replace the John-Nirenberg space $B M O$ (in the classical result) by a new $B M O_{L}$, associated to a semigroup of operators $\left(e^{-t L}\right)_{t>0}$. These new spaces $B M O_{L}$ (including $B M O$ ) have recently appeared in numerous works in order to extend the theory of Hardy and $B M O$ space to more general situations. Then we give applications by describing boundedness for a new kind of paraproduct, built on the considered semigroup. In addition we obtain a version of the classical $T(1)$-theorem for doubling Riemannian manifolds.
\end{abstract}

\section{Contents}

1. Introduction

2. Preliminaries

2.1. The doubling property

2.2. Poincaré inequality

2.3. Framework for semigroup of operators

6076

2.4. BMO space

6078

2.5. Examples of such semigroups

6079

2.5.1. Second-order elliptic operator

2.5.2. Laplacian operators on a manifold 6079

3. A $T(1)$-theorem for semigroups $\quad 6080$

3.1. Assumptions and statement $\quad 6080$

3.2. Proof of Theorem 3.3

4. Applications to new paraproducts and to Calderón-Zygmund operators

on a Riemannian manifold 6095

4.1. Boundedness of new paraproducts in Lebesgue spaces with $r^{\prime}>1 \quad 6097$

4.2. Boundedness in weighted Lebesgue spaces and extrapolation to the case $r^{\prime} \leq 1$

4.3. A "classical" $T(1)$-theorem on a Riemannian manifold 6104

References

Received by the editors April 22, 2010 and, in revised form, April 25, 2011.

2010 Mathematics Subject Classification. Primary 30E20, 42B20, 42B30 .

Key words and phrases. T(1)-theorem, semigroup of operators, paraproducts.

(C)2012 American Mathematical Society Reverts to public domain 28 years from publication 


\section{INTRODUCTION}

The T(1)-theorem of G. David and J. L. Journé provides a very powerful tool for analyzing the $L^{2}$-boundedness of Calderón-Zygmund. It claims that for such a linear operator $T$, if it satisfies a weak boundedness property, then $T$ is bounded on $L^{2}$ if and only if $T(1)$ and $T^{*}(1)$ belong to the John-Nirenberg space $B M O$ (introduced in [39]). This result, proved in the Euclidean space framework in [20, is based on an appropriate reproducing Calderón formula and the notion of Carleson measure (closely related to the space $B M O$ ). It permits us, for example, to obtain a new proof of the $L^{2}$-boundedness of the first Calderón commutator and to obtain a characterization of the $L^{2}$-bounded pseudo-differential operators (belonging to some exotic class such as $S_{1,1}^{0}$; see [15).

Then this result was extended by F. Nazarov, S. Treil and A. Volberg for a non-doubling measure in [47] and by X. Tolsa in proper subsets of the Euclidean space (not satisfying the doubling property; see [52]). Then many works deal with the following problem: change the function 1 by an accretive function $b$ getting the so-called "T(b)-theorem" (see the work of A. McIntosh and Y. Meyer 43 and of G. David, J.L Journé and S. Semmes 21]) or by a system of accretive functions, obtaining the so-called "local $T(b)$-theorem" (see the work of M. Christ 14 and other works such as 48). We refer the reader to a survey of S. Hofmann 35] about such questions and related applications to PDEs. Numerous works deal with some adaptations, for example to quadratic T(1)-type theorems (see [2]), to offdiagonal T(1)-theorems (see [34]), to Triebel-Lizorkin spaces (see [54] and [53]) or to vector-valued operators (see [37, 38]).

All these results concern Calderón-Zygmund operators and involve the JohnNirenberg space $B M O$. This space naturally arises as the class of functions whose deviation from their means over cubes is bounded. This space strictly includes the $L^{\infty}$ space and is a good extension of the Lebesgue spaces scale $\left(L^{p}\right)_{1<p<\infty}$ for $p \rightarrow$ $\infty$ from the point of view of harmonic analysis. For example, it plays an important role in boundedness of Calderón-Zygmund operators, real interpolation, Carleson measure, and the study of paraproducts. Unfortunately, there are situations where the John-Nirenberg space $B M O$ is not the right substitute to $L^{\infty}$ and there have recently been numerous works whose goal is to define an adapted $B M O$ space according to the context (see [23, 24, 36]). For example the classical space $B M O$ is not well adapted to operators such as the Riesz transform on Riemannian manifolds. That is why in 23] and 24, X. T. Duong and L. Yan studied some new BMO type spaces associated to a semigroup (satisfying pointwise bounds) and proved an associated version of the John-Nirenberg inequality on these spaces, duality and interpolation results. Then, in [36], this theory was extended by S. Hofmann and S. Mayboroda to a setting in which pointwise kernel bounds may be lacking. We direct the reader to the work of P. Auscher, A. McIntosh and E. Russ [6], where much of the theory of 36 was developed independently, albeit in a somewhat different setting.

In the recent works [10, 12, J. Zhao and the author have developed an abstract framework for $B M O$ spaces (and proved some results about John-Nirenberg inequalities). This framework permits us to cover the classical space $B M O$ and those defined in 24] and 36.

The aim of this article is to continue the study of $T(1)$-theorems using these new spaces $B M O$, defined by a semigroup of operators. 
Let us just describe some motivation to such results. Associated to the $B M O$ space, which is a good extension of the Lebesgue spaces scale $\left(L^{p}\right)_{1<p<\infty}$ for $p \rightarrow \infty$, there are the "Hardy spaces" $H^{1}$ for $p \rightarrow 1$. On the Euclidean space, R. Coifman and G. Weiss have introduced the first Hardy space via atomic decomposition in 17. Then several characterizations were obtained in [51, via the maximal function or Riesz transform. The equivalence between all these definitions due to R. Coifman in 29] can be understood from the celebrated theorem of C. Fefferman which says

$$
\left(H^{1}\right)^{*}=B M O
$$

As $B M O$ for $L^{\infty}$, the space $H^{1}$ is a good substitute of $L^{1}(X)$ for many reasons. For instance, Calderón-Zygmund operators map $H^{1}$ to $L^{1}$. In addition, $H^{1}$ (and its dual) interpolates with Lebesgue spaces $L^{p}, 1<p<\infty$. However, there are situations where the space $H^{1}$ is not the right substitute for $L^{1}$ and there has recently been a number of works with the goal of defining an adapted Hardy space [3, 4, 8, 10, 11, 24, 23, 25, 26, 27, 28, 36. In [10, 11, the authors have described a very abstract theory for Hardy spaces (built via atomic decomposition) and interpolation results with Lebesgue spaces. This part seems to be well understood. In particular concerning Hardy spaces associated to a semigroup, we have several characterizations via atomic decomposition, the area square function and the maximal function.

In order to pursue this theory, it is now important to get criteria for an operator to be bounded in $L^{2}$. Then using this theory of Hardy spaces, we know how to obtain $L^{p}$-boundedness from the initial $L^{2}$-boundedness. Aiming at that, we are motivated to obtain a general $T(1)$-theorem (associated to this framework of semigroups).

So consider an operator $L$ of order $m$ acting on a doubling Riemannian manifold $(M, d, \mu)$, such that it admits a holomorphic calculus. In this case, we can consider the semigroup $\left(e^{-t L}\right)_{t>0}$. We define the space $B M O_{L}$ as the set of functions $f$ such that

$$
\sup _{t>0} \sup _{\substack{Q \text { ball } \\ r_{Q}^{m}=t}} \frac{1}{\mu(Q)} \int_{Q}\left|f-e^{-t L} f\right| d \mu<\infty .
$$

We refer the reader to Section 2 for precise assumptions on the manifold and on the semigroup and preliminaries on this new $B M O$ space. Then our main theorem is the following one (see Theorem 3.3):

Theorem 1.1. Let $T$ be a linear operator, weakly continuous on $L^{2}(M)$ and admitting off-diagonal decays relative to cancellation built with the semigroup (see Assumptions (3.1), (3.2) and (3.3)). Then if $T(1) \in B M O_{L}$ and $T^{*}(1) \in B M O_{L^{*}}$, then $T$ admits a bounded extension in $L^{2}(M)$.

Moreover we will describe a reverse property: if $T$ admits an $L^{2}$-bounded extension, then $T(1)$ and $T^{*}(1)$ belong to some $B M O$ spaces (closely related to $B M O_{L}$ and $\left.B M O_{L^{*}}\right)$.

So we obtain as for the original theorem of G. David and J.L. Journé, a criterion for the $L^{2}$-boundedness via these new spaces $B M O_{L}$. We emphasize that assumptions (3.1), (3.2) and (3.3) can just be considered as generalizations of the 
usual Calderón-Zygmund properties since if we consider $L=-\Delta$ on $\mathbb{R}^{d}$, then every Calderón-Zygmund operator satisfies (3.1), (3.2) and (3.3). So we recover the original $T(1)$-theorem (since $B M O_{L}=B M O$ in this case).

Then in Section 4 we apply this new $T(1)$-theorem to a new kind of paraproduct. In the usual framework, paraproducts were introduced by J. M. Bony [13] and then studied by Y. Meyer in [45, 46]. These operators are considered as the prototype of the so-called "Coifman-Meyer bilinear operators", which are particular bilinear Calderón-Zygmund. A main result says that they are bounded from $L^{p} \times L^{q}$ into $L^{r^{\prime}}$ as soon as $1<p, q \leq \infty$ and

$$
\frac{1}{p}+\frac{1}{q}=\frac{1}{r^{\prime}} .
$$

These operators appear in the study of the pointwise product between two functions and their study requires understanding the frequency analysis of the product. Here, we introduce new bilinear operators, built with semigroups, which correspond to paraproducts with a frequency analysis adapted to the spectral properties of our semigroups $e^{-t L}$. In this case, it is not clear how $e^{-t L}$ acts on a product of two functions and that is why the boundedness of such operators is not clear (see Remark 4.4). We refer the reader to Section 4 for a precise statement of the results. We prove the following one:

Theorem 1.2 (Theorem 4.10). Let $\psi$ and $\phi$ be defined as in Theorems 4.5 and 4.6. Let $p, q \in(1, \infty)$ and $r^{\prime} \in(1 / 2, \infty)$ be exponents satisfying

$$
\frac{1}{p}+\frac{1}{q}=\frac{1}{r^{\prime}} .
$$

The paraproducts

$$
(f, h) \rightarrow \int_{0}^{\infty} \psi_{t}(L)\left[\phi_{t}(L) f \phi_{t}(L) h\right] \frac{d t}{t}
$$

and

$$
(h, f) \rightarrow \int_{0}^{\infty} \phi_{t}(L)\left[\psi_{t}(L) f \phi_{t}(L) h\right] \frac{d t}{t}
$$

are bounded from $L^{p}(M) \times L^{q}(M)$ to $L^{r^{\prime}}(M)$.

Moreover we obtain weighted estimates.

We finish this work by describing, in Subsection 4.3 a version of the classical $T(1)$-theorem for Calderón-Zygmund operators in general doubling Riemannian manifolds. All the previous cited works only deal with the Euclidean space and are based on some specific differential properties of $\mathbb{R}^{n}$. That is why it seems important to us to prove such a result, and we obtain it as an application of our main result.

\section{Preliminaries}

For a ball $Q$ in a metric space, $\lambda Q$ denotes the ball co-centered with $Q$ and with radius $\lambda$ times that of $Q$. Finally, $C$ will be a constant that may change from one inequality to another, and we will use $u \lesssim v$ to say that there exists a constant $C$ such that $u \leq C v$ and use $u \simeq v$ to say that $u \lesssim v$ and $v \lesssim u$.

In all of this paper, $M$ denotes a complete Riemannian manifold. We write $\mu$ for the Riemannian measure on $M, \nabla$ for the Riemannian gradient, $|\cdot|$ for the length on the tangent space (forgetting the subscript $x$ for simplicity) and $\|\cdot\|_{L^{p}}$ for the 
norm on $L^{p}:=L^{p}(M, \mu), 1 \leq p \leq+\infty$. We denote by $Q(x, r)$ the open ball of center $x \in M$ and radius $r>0$.

We deal with the Sobolev spaces of order $1, W^{1, p}:=W^{1, p}(M)$, where the norm is defined by:

$$
\|f\|_{W^{1, p}(M)}:=\|f\|_{p}+\||\nabla f|\|_{L^{p}} .
$$

We write $\mathcal{S}(M)$ for the Schwartz space on the manifold $M$ and $\mathcal{S}^{\prime}(M)$ for its dual, corresponding to the set of distributions. Moreover in all of this work, $\mathbf{1}=\mathbf{1}_{M}$ will be used for the constant function that is equal to one on the whole manifold.

\subsection{The doubling property.}

Definition 2.1 (Doubling property). Let $M$ be a Riemannian manifold. One says that $M$ satisfies the doubling property $(D)$ if there exists a constant $C_{0}>0$, such that for all $x \in M, r>0$ we have

$$
\mu(B(x, 2 r)) \leq C_{0} \mu(B(x, r)) .
$$

Lemma 2.2. Let $M$ be a Riemannian manifold satisfying $(D)$ and let $d:=\log _{2} C_{0}$. Then for all $x, y \in M$ and $\theta \geq 1$,

$$
\mu(B(x, \theta R)) \leq C \theta^{d} \mu(B(x, R)) .
$$

There also exists $c$ and $N \geq 0$, so that for all $x, y \in M$ and $r>0$,

$$
\mu(B(y, r)) \leq c\left(1+\frac{d(x, y)}{r}\right)^{N} \mu(B(x, r)) .
$$

For example, if $M$ is the Euclidean space $M=\mathbb{R}^{d}$, then $N=0$ and $c=1$.

Observe that if $M$ satisfies $(D)$, then

$$
\operatorname{diam}(M)<\infty \Leftrightarrow \mu(M)<\infty \text { (see [1]). }
$$

Therefore if $M$ is a complete Riemannian manifold satisfying $(D)$, then $\mu(M)=\infty$.

Theorem 2.3 (Maximal theorem [16]). Let $M$ be a Riemannian manifold satisfying $(D)$. Denote by $\mathcal{M}$ the uncentered Hardy-Littlewood maximal function over open balls of $M$ defined by

$$
\mathcal{M} f(x):=\sup _{\substack{B \text { ball } \\ x \in B}} \frac{1}{\mu(B)} \int_{B}|f| d \mu .
$$

Then for every $p \in(1, \infty], \mathcal{M}$ is $L^{p}$-bounded and moreover of weak type $(1,1)[1$

Consequently for $s \in(0, \infty)$, the operator $\mathcal{M}_{s}$ defined by

$$
\mathcal{M}_{s} f(x):=\left[\mathcal{M}\left(|f|^{s}\right)(x)\right]^{1 / s}
$$

is of weak type $(s, s)$ and $L^{p}$ bounded for all $p \in(s, \infty]$.

\footnotetext{
${ }^{1}$ An operator $T$ is of weak type $(p, p)$ if there is $C>0$ such that for any $\alpha>0, \mu(\{x$ : $|T f(x)|>\alpha\}) \leq \frac{C}{\alpha^{p}}\|f\|_{p}^{p}$
} 


\subsection{Poincaré inequality.}

Definition 2.4 (Poincaré inequality on $M$ ). We say that a complete Riemannian manifold $M$ admits a Poincaré inequality $\left(P_{q}\right)$ for some $q \in[1, \infty)$ if there exists a constant $C>0$ such that, for every function $f \in W_{l o c}^{1, q}(M)$ (the set of compactly supported Lipschitz functions on $M$ ) and every ball $B$ of $M$ of radius $r>0$, we have

$$
\left(f_{B}\left|f-f_{B} f d \mu\right|^{q} d \mu\right)^{1 / q} \leq C r\left(f_{B}|\nabla f|^{q} d \mu\right)^{1 / q} .
$$

Remark 2.5. By density of $C_{0}^{\infty}(M)$ in $W_{l o c}^{1, q}(M)$, we can replace $W_{l o c}^{1, q}(M)$ by $C_{0}^{\infty}(M)$.

Let us recall some known facts about Poincaré inequalities with varying $q$.

It is known that $\left(P_{q}\right)$ implies $\left(P_{p}\right)$ when $p \geq q$ (see [33]). Thus, if the set of $q$ such that $\left(P_{q}\right)$ holds is not empty, then it is an interval unbounded on the right. A recent result of S. Keith and X. Zhong (see [40) asserts that this interval is open in $[1,+\infty[$ :

Theorem 2.6. Let $(M, d, \mu)$ be a doubling and complete Riemannian manifold, admitting a Poincaré inequality $\left(P_{q}\right)$, for some $1<q<\infty$. Then there exists $\epsilon>0$ such that $(M, d, \mu)$ admits $\left(P_{p}\right)$ for every $p>q-\epsilon$.

We refer the reader to Theorem 5.3.3 of [49] or Proposition 1.6 of 9] for the proof of the following consequence.

Proposition 2.7. Assume that $M$ satisfies $(D)$ and admits a Poincaré inequality $\left(P_{q}\right)$ for some $q \in[1, \infty)$. Then there is a constant $c=c(q)$ and $\epsilon>0$ such that for all function $f \in W_{l o c}^{1, q}$,

$$
|f(x)-f(y)| \leq c d(x, y)\left[\mathcal{M}_{q-\epsilon}(|\nabla f|)(x)+\mathcal{M}_{q-\epsilon}(|\nabla f|)(y)\right] .
$$

2.3. Framework for semigroup of operators. Let us recall the framework of 23, 24.

Let $\omega \in[0, \pi / 2)$. We define the closed sector in the complex plane $\mathbb{C}$ by

$$
S_{\omega}:=\{z \in \mathbb{C}:|\arg (z)| \leq \omega\} \cup\{0\}
$$

and denote the interior of $S_{\omega}$ by $S_{\omega}^{0}$. We use the notation $H_{\infty}\left(S_{\omega}^{0}\right)$ for the set of bounded holomorphic functions $b$ on $S_{\omega}^{0}$, equipped with the norm

$$
\|b\|_{H_{\infty}\left(S_{\omega}^{0}\right)}:=\|b\|_{L^{\infty}\left(S_{\omega}^{0}\right)} .
$$

Then consider a linear operator $L$. It is said to be of type $\omega$ if its spectrum $\sigma(L) \subset S_{\omega}$, and for each $\nu>\omega$ there exists a constant $c_{\nu}$ such that

$$
\left\|(L-\lambda)^{-1}\right\|_{L^{2} \rightarrow L^{2}} \leq c_{\nu}|\lambda|^{-1}
$$

for all $\lambda \notin S_{\nu}$.

We refer the reader to [23] and 42 for more details concerning the holomorphic calculus of such operators. In particular, it is well known that $L$ generates a holomorphic semigroup $\left(\mathcal{A}_{z}:=e^{-z L}\right)_{z \in S_{\pi / 2-\omega}}$. Let us now detail some assumptions that we make on the semigroup. 
Assume the following conditions: there exist a positive real $m>0$ and $\delta>1$ with 2

- For every $z \in S_{\pi / 2-\omega}$, the linear operator $\mathcal{A}_{z}:=e^{-z L}$ is given by a kernel $a_{z}$ satisfying

$$
\left|a_{z}(x, y)\right| \lesssim \frac{1}{\mu\left(B\left(x,|z|^{1 / m}\right)\right)}\left(1+\frac{d(x, y)}{|z|^{1 / m}}\right)^{-d-2 N-\delta},
$$

where $d$ is the homogeneous dimension of the space (see (2.1)) and $N$ is the other dimension parameter (see (2.2) $) ; N \geq 0$ could be equal to 0 .

- The operator $L$ has a bounded $H_{\infty}$-calculus on $L^{2}$. That is, there exists $c_{\nu}$ such that for $b \in H_{\infty}\left(S_{\nu}^{0}\right)$, we can define $b(L)$ as an $L^{2}$-bounded linear operator and

$$
\|b(L)\|_{L^{2} \rightarrow L^{2}} \leq c_{\nu}\|b\|_{\infty} .
$$

Remark 2.8. The assumed bounded $H_{\infty}$-calculus on $L^{2}$ allows us to deduce some extra properties (see 24] and [42]):

- Due to the Cauchy formula for complex differentiation, the pointwise estimate (2.4) still holds for the kernel of $(t L)^{k} e^{-t L}$ with $t>0$ and $k \in \mathbb{N}$.

- For any holomorphic function $\psi \in H\left(S_{\nu}^{0}\right)$ such that for some $s>0,|\psi(z)| \lesssim$ $\frac{|z|^{s}}{1+|z|^{2 s}}$, the quadratic functional

$$
f \rightarrow\left(\int_{0}^{\infty}|\psi(t L) f|^{2} \frac{d t}{t}\right)^{1 / 2}
$$

is $L^{2}$-bounded.

Moreover we need another assumption on the semigroup, which concerns a square estimate on the gradient of the semigroup. We assume that for every integer $k \geq 0$, the square functional

$$
f \rightarrow\left(\int_{0}^{\infty}\left|t^{1 / m} \nabla(t L)^{k} e^{-t L}(f)\right|^{2} \frac{d t}{t}\right)^{1 / 2}
$$

is bounded on $L^{2}$.

It is interesting to observe that except for (2.6), we do not require regularity assumptions on the heat kernel.

Remark 2.9. We claim that assumption (2.6) is satisfied if the Riesz transform $\mathcal{R}:=\nabla L^{-1 / m}$ is $L^{2}$-bounded.

Indeed if $\mathcal{R}$ is $L^{2}$-bounded, then it admits $l^{2}$-valued estimates, which yields

$$
\begin{aligned}
\left(\int_{M} \int_{0}^{\infty}\right. & \left.\left|\mathcal{R}(t L)^{k+1 / m} e^{-t L}(f)\right|^{2} \frac{d t d \mu}{t}\right)^{1 / 2} \\
& \leq\|\mathcal{R}\|_{L^{2} \rightarrow L^{2}}\left(\int_{M} \int_{0}^{\infty}\left|(t L)^{k+1 / m} e^{-t L}(f)\right|^{2} \frac{d t d \mu}{t}\right)^{1 / 2} .
\end{aligned}
$$

\footnotetext{
${ }^{2}$ Usually, see (23 24] , we just require that $\delta>0$. In our work, we will use the Poincaré inequality and so we need to compensate for a power 1 of the distance. That is why we require $\delta>1$.
} 
This gives the desired result,

$$
\left(\int_{M} \int_{0}^{\infty}\left|t^{1 / m} \nabla(t L)^{k} e^{-t L}(f)\right|^{2} \frac{d t d \mu}{t}\right)^{1 / 2} \lesssim\|f\|_{L^{2}},
$$

thanks to Remark 2.8 ,

Remark 2.10. Using Stein's complex interpolation theorem, it is known that the boundedness of

$$
f \rightarrow\left(\int_{0}^{\infty}\left|s^{1 / m} \nabla e^{-s L}(f)\right|^{2} \frac{d t}{t}\right)^{1 / 2}
$$

on $L^{p}$ for every $p$ belonging to a neighborhood of $2 \in(1, \infty)$ implies the $L^{2}$ boundedness of (2.6) for $k \in \mathbb{N}$. We refer the reader to Step 7 of the proof of Theorem 6.1 in 4 for a detailed proof of such a result.

2.4. BMO space. According to 24, it is well known that BMO spaces related to semigroups are well defined as a subspace of

$$
\mathbb{M}:=\bigcup_{x_{0} \in M} \bigcup_{\beta \in(0, \delta)} \mathbb{M}_{x_{0}, \beta}
$$

with $\mathbb{M}_{x_{0}, \beta}$ the set of functions $f \in L_{l o c}^{1}$ such that

$$
\|f\|_{\mathbb{M}_{x_{0}, \beta}}:=\int \frac{|f(x)|}{\left(1+d\left(x_{0}, x\right)\right)^{2 N+\beta} \mu\left(B\left(x_{0}, 1+d\left(x_{0}, x\right)\right)\right)} d \mu(x)<\infty,
$$

where $N$ is given by (2.2) and $\delta$ by (2.4).

Definition 2.11 (Definition 2.4 [24]). A function $f \in \mathbb{M}$ belongs to $B M O_{L}$ if and only if

$$
\|f\|_{B M O_{L}}:=\sup _{t>0} \sup _{\substack{B \text { ball } \\ r_{B}^{\text {mall }}}} \frac{1}{\mu(B)} \int_{B}\left|f-e^{-t L} f\right| d \mu<\infty .
$$

We refer to 23 and 24 for a study of precise examples concerning this kind of $B M O$ spaces. We underline that $B M O_{L}$ satisfies some John-Nirenberg properties and we now state some useful properties:

Proposition 2.12 (Theorem 2.14 [24]). For any $f \in B M O_{L}$, the measure

$$
d \nu(x, t):=\left|t^{m} L e^{-t^{m} L}\left(1-e^{-t^{m} L}\right)\right|^{2} \frac{d \mu(x) d t}{t}
$$

is a Carleson measure. Moreover for all integers $k \geq 1$ the measure

$$
d \nu_{k}(x, t):=\left|\left(t^{m} L\right)^{k} e^{-t^{m} L}\left(1-e^{-t^{m} L}\right)\right|^{2} \frac{d \mu(x) d t}{t}
$$

is a Carleson measure too.

Proof. In [24, the proof is only explained for the Laplacian operator $L=-\Delta$ in the Euclidean space with $k=1$. However, the authors used technical properties on $B M O$ spaces, which are detailed and proved in the general framework (for example the John-Nirenberg inequality). In addition the proof relies on the $L^{2}$-boundedness of the square function

$$
\left(\int_{0}^{\infty}\left|\left(t^{m} L\right)^{k} e^{-t^{m} L}\left(1-e^{-t^{m} L}\right) f\right|^{2} \frac{d t}{t}\right)^{1 / 2},
$$

which is a consequence of assumption (2.5); see Remark 2.8, The allowed integer $k \geq 1$ has already appeared in Theorem 9.1 of [36] in a particular context. 
Remark 2.13. In our main result, Theorem 3.3. we require the extra assumption

$$
L(\mathbf{1})=L^{*}(\mathbf{1})=0 .
$$

Under this assumption, it is known that the classical space $B M O$ is included in the new one:

$$
B M O \hookrightarrow B M O_{L}
$$

see Proposition 6.7 [23] and Remark 7.6 of [10 for a more general study of this question. In addition, this inclusion may be strict; we refer the reader to Proposition 6.823 for an example.

2.5. Examples of such semigroups. In this subsection, we would like to give two examples of situations where all these assumptions are satisfied.

2.5.1. Second-order elliptic operator. Let $M=\mathbb{R}^{d}$ and $A$ be a $d \times d$ matrix-valued function satisfying the ellipticity condition: there exist two constants $\Lambda \geq \lambda>0$ such that

$$
\forall \xi, \zeta \in \mathbb{C}^{d}, \quad \lambda|\xi|^{2} \leq \operatorname{Re}(A \xi \cdot \bar{\xi}) \quad \text { and } \quad|A \xi \cdot \bar{\zeta}| \leq \Lambda|\xi||\zeta| .
$$

We define the second-order divergence form operator

$$
L(f):=-\operatorname{div}(A \nabla f) .
$$

This particular framework was studied for example by P. Auscher in [4 and by S. Hofmann and S. Mayboroda in [36. We define the second-order divergence form operator $L(f)=-\operatorname{div}(A \nabla f)$, which can be interpreted in the weak sense via sesquilinear forms. Since $L$ is maximal accretive, it admits a bounded $H_{\infty}$-calculus on $L^{2}\left(\mathbb{R}^{d}\right)$. Moreover when $A$ has real entries or when the dimension $d \in\{1,2\}$, then the operator $L$ generates an analytic semigroup on $L^{2}$ with a heat kernel satisfying Gaussian upper bounds. In this case all our assumptions are verified with $m=2$ (see [4 for estimates on the gradient of the semigroup).

2.5.2. Laplacian operators on a manifold. Let $M$ be a doubling connected noncompact Riemannian manifold and consider $L=-\Delta$ to be the positive LaplaceBeltrami operator. Let $p_{t}$ be the heat kernel of $e^{-t L}$. It is well known that ondiagonal upper bound

$$
\sup _{x \in M}\left|p_{t}(x, x)\right| \lesssim t^{-d / 2}
$$

self-improves into a Gaussian off-diagonal bound (which implies (2.4) with $m=2$ ). This can be seen in several ways, by using a perturbation method of Davies, or the integrated maximum principle, or the finite propagation speed of solutions to the wave equation (see [18, 32, 49, 50). Moreover, we know that (2.8) implies the $L^{p}$-boundedness of the Riesz transform for every $p \in(1,2]$ (see Theorem 1.1 [19]).

In addition, by integration by parts we easily obtain the $L^{2}$-boundedness of the Riesz transform and so (2.6) is verified, thanks to Remark 2.9. We refer the reader to [5] for more details concerning these kinds of assumptions and how they are related between them.

Concerning the extension to a complex semigroup, we refer the reader to 22 . (Lemma 2). Since $L$ is a non-negative self-adjoint operator, the semigroup $e^{-z L}$ is holomorphic in $S_{\pi / 2}$ and $L$ admits an $H_{\infty}$-bounded holomorphic calculus.

Consequently, for example, if the manifold $M$ has non-negative Ricci curvature, all our assumptions are satisfied. 


\section{A T(1)-THEOREM FOR SEMIGROUPS}

3.1. Assumptions and statement. Before stating our T(1)-theorem, we have to assume some properties on a generic operator $T$ (in order to replace the usual Calderón-Zygmund properties on the kernel in the classical $T(1)$-theorem). Let $T$ be a continuous operator acting from $\mathcal{S}(M)$ into $\mathcal{S}^{\prime}(M)$. We assume that $T$ and $T^{*}$ satisfy some $L^{2}-L^{2}$ off-diagonal decay as follows: there exists an integer $\kappa \geq 1$ such that for every $s>0$, every ball $Q_{1}, Q_{2}$ of radius $r:=s^{1 / m}$ and function $f \in L^{2}\left(Q_{1}\right)$,

- if $d\left(Q_{1}, Q_{2}\right) \geq 2 r$, then we have

$$
\left\|(s L)^{\kappa} e^{-s L} T(f)\right\|_{L^{2}\left(Q_{2}\right)} \lesssim\left(1+\frac{d\left(Q_{1}, Q_{2}\right)}{r}\right)^{-d-2 N-\delta}\|f\|_{L^{2}\left(Q_{1}\right)}
$$

and the dual estimates

$$
\left\|\left(s L^{*}\right)^{\kappa} e^{-s L^{*}} T^{*}(f)\right\|_{L^{2}\left(Q_{2}\right)} \lesssim\left(1+\frac{d\left(Q_{1}, Q_{2}\right)}{r}\right)^{-d-2 N-\delta}\|f\|_{L^{2}\left(Q_{1}\right)} ;
$$

- if $d\left(Q_{1}, Q_{2}\right) \leq 2 r$, then we have

$$
\left\|(s L)^{\kappa} e^{-s L} T\left(e^{-s L} f\right)\right\|_{L^{2}\left(Q_{2}\right)}+\left\|\left(s L^{*}\right)^{\kappa} e^{-s L^{*}} T\left(e^{-s L^{*}} f\right)\right\|_{L^{2}\left(Q_{2}\right)} \lesssim\|f\|_{L^{2}\left(Q_{1}\right)} .
$$

We call this property the weak boundedness of $T$.

Remark 3.1. We claim that (3.3) self-improves in the following case: for all integers $k \geq 0\left(\right.$ with $\left.d\left(Q_{1}, Q_{2}\right) \leq 2 r\right)$

$$
\begin{aligned}
\left\|(s L)^{\kappa} e^{-s L} T\left((s L)^{k} e^{-s L} f\right)\right\|_{L^{2}\left(Q_{2}\right)}+\left\|\left(s L^{*}\right)^{\kappa} e^{-s L^{*}} T\left((s L)^{k} e^{-s L} f\right)\right\|_{L^{2}\left(Q_{2}\right)} & \\
& \lesssim\|f\|_{L^{2}\left(Q_{1}\right)} .
\end{aligned}
$$

Let us explain how we can obtain this improvement for $T$. We choose a bounded covering $\left(R_{l}\right)_{l}$ of the manifold by balls of radius $r:=(s / 2)^{1 / m}$. Applying (3.3) and (3.1) to the function $(s L)^{k} e^{-s L / 2}(f)$, we get

$$
\begin{aligned}
\left\|(s L)^{\kappa} e^{-s L} T\left((s L)^{k} e^{-s L} f\right)\right\|_{L^{2}\left(Q_{2}\right)} & \\
& \lesssim \sum_{l}\left(1+\frac{d\left(Q_{2}, R_{l}\right)}{r}\right)^{-d-2 N-\delta}\left\|(s L)^{k} e^{-s L / 2}(f)\right\|_{L^{2}\left(R_{l}\right)} .
\end{aligned}
$$

Then, due to the off-diagonal decay of the derivative of the semigroup, we have

$$
\begin{aligned}
\left\|(s L)^{\kappa} e^{-s L} T\left((s L)^{k} e^{-s L} f\right)\right\|_{L^{2}\left(Q_{2}\right)} & \\
& \lesssim \sum_{l}\left(1+\frac{d\left(Q_{2}, R_{l}\right)}{r}\right)^{-d-2 N-\delta}\left(1+\frac{d\left(Q_{1}, R_{l}\right)}{r}\right)^{-d-2 N-\delta}\|f\|_{L^{2}\left(Q_{1}\right)} \\
& \lesssim\|f\|_{L^{2}\left(Q_{1}\right)} .
\end{aligned}
$$

Remark 3.2. The weak boundedness property of the operator $T$ is obviously a necessary condition for the $L^{2}$-boundedness.

Theorem 3.3. Assume that the Riemannian manifold $M$ satisfies the Poincaré inequality $\left(P_{2}\right)$ and has an infinite measure $\mu(M)=\infty$. Suppose the existence of an operator $L$ such that $L(\mathbf{1})=0=L^{*}(\mathbf{1})$ and such that the corresponding semigroup 
satisfies the assumptions of Subsection 2.3. Let T be a continuous operator weakly continuous from $L^{2}$ into $L^{2}$ satisfying (3.1), (3.2) and (3.3).

- If $T(\mathbf{1}) \in B M O_{L}$ and $T^{*}(\mathbf{1}) \in B M O_{L^{*}}$, then $T$ admits an $L^{2}$-bounded extension. Moreover $\|T\|_{L^{2} \rightarrow L^{2}}$ is only controlled by the implicit constants and $\|T(\mathbf{1})\|_{B M O_{L}}+\left\|T^{*}(\mathbf{1})\right\|_{B M O_{L^{*}}}$ (and not by the weak continuity from $L^{2}$ to $\left.L^{2}\right)$.

- Let us assume $\kappa=1$ in (3.1), (3.2) and (3.3). If $T$ admits a continuous extension on $L^{2}$, then $T(\mathbf{1})$ belongs to $B M O_{L}$ and $T^{*}(\mathbf{1})$ to $B M O_{L^{*}}$.

Remark 3.4. In the previous statement, we implicitly assume that $T(\mathbf{1})$ and $T^{*}(\mathbf{1})$ are well defined and belong to $\mathbb{M}$. We do not deal with this specific problem as in our applications, we can work with some kind of "truncations" $T_{\epsilon}$ of $T$, where $T_{\epsilon}(\mathbf{1})$ and $T_{\epsilon}^{*}(\mathbf{1})$ will be well defined.

Remark 3.5. For the second part of the theorem, for another integer $\kappa \geq 1$, if $T$ admits a continuous extension on $L^{2}$, then $T(\mathbf{1})$ and $T^{*}(\mathbf{1})$ belong to another kind of $B M O_{L}$ space (which requires more cancellation) and satisfies

$$
\sup _{t>0} \sup _{\substack{Q \text { ball } \\ r_{Q}^{m}=t}} \frac{1}{\mu(Q)} \int_{Q}\left|\left(1-e^{-t L}\right)^{\kappa} T(\mathbf{1})\right| d \mu<\infty
$$

and

$$
\sup _{t>0} \sup _{\substack{Q \text { ball } \\ r_{Q}=t}} \frac{1}{\mu(Q)} \int_{Q}\left|\left(1-e^{-t L^{*}}\right)^{\kappa} T^{*}(\mathbf{1})\right| d \mu<\infty .
$$

We leave it to the reader to check this claim by adapting the proof made for the second part of Theorem 3.2.

Corollary 3.6. Under the assumptions of Theorem 3.3, for all exponents $p \in$ $(1, \infty)$ there is a constant $c_{p}$ such that

$$
\forall f \in \mathcal{S}, \quad\|T(f)\|_{L^{p}} \leq c_{p}\|f\|_{L^{p}} .
$$

Proof. From Theorem [3.3, we know that the operator $T$ admits a continuous extension in $L^{2}$. To deduce an $L^{p}$-boundedness from an $L^{2}$-boundedness, we use the theory of Hardy spaces.

More precisely, we refer to [10] for an abstract theory of Hardy spaces, which we are going to apply. We have to build an adapted Hardy space $H_{L}^{1}$ (Step 1), then prove that our operator $T$ is bounded from $H_{L}^{1}$ to $L^{1}$ (Step 2) and then interpolate the Hardy space with $L^{2}$ (Step 3).

Step 1. Construction of an adapted Hardy space.

For $Q$ a ball of radius $r=s^{1 / m}$, we define the operator

$$
A_{Q}:=1-\left(1-e^{-s L}\right)^{\kappa} .
$$

These operators are uniformly bounded on $L^{2}$ (since they correspond to a finite sum of semigroups). We refer the reader to [10] for an abstract construction of a Hardy space $H_{L}^{1}$, based on a collection of operators $\left(A_{Q}\right)_{Q}$, indexed by the balls. We refer to 23. for more specific results concerning the particular case where $A_{Q}$ is defined by a semigroup. Indeed, the Hardy space is defined by atomic decomposition. By definition for a ball $Q$, an atom (relative to the ball $Q$ ) is a function $m=f-A_{Q}(f)$, where $f \in L^{2}(Q)$ is $L^{1}$-normalized in $L^{2}$. 
Step 2. Boundedness of $T$ from $H_{L}^{1}$ to $L^{1}$.

To check that $T$ is bounded on the implicit Hardy space $H_{L}^{1}$, it just suffices to prove some $L^{2}-L^{2}$ off-diagonal decay (see Theorem 4.2 of [10]): for every ball $Q$ or radius $r=s^{1 / m}$ and integer $i \geq 1$,

$$
\left(\frac{1}{\mu\left(2^{i} Q\right)} \int_{2^{i} Q \backslash 2^{i-1} Q}\left|T\left(1-e^{-s L}\right)^{\kappa}(f)\right|^{2} d \mu\right)^{1 / 2} \lesssim \gamma(i)\|f\|_{L^{2}(Q)}
$$

with fast decreasing coefficients $\gamma(i)$ such that

$$
\sum_{i} 2^{i d} \gamma(i)<\infty
$$

and

$$
\left(\frac{1}{\mu(2 Q)} \int_{2 Q}\left|T\left(1-e^{-s L}\right)^{\kappa}(f)\right|^{2} d \mu\right)^{1 / 2} \lesssim\|f\|_{L^{2}(Q)} .
$$

These off-diagonal estimates are a consequence of (3.2). Indeed the off-diagonal decay (3.2) can be improved as follows: for every $s>0$, all balls $Q_{1}, Q_{2}$ of radius $r:=s^{1 / m}$ and functions $f \in L^{2}\left(Q_{1}\right)$, then we have

$$
\left\|\left(s L^{*}\right)^{\kappa} e^{-s L^{*}} T^{*}(f)\right\|_{L^{2}\left(Q_{2}\right)} \lesssim\left(1+\frac{d\left(Q_{1}, Q_{2}\right)}{r}\right)^{-d-2 N-\delta}\|f\|_{L^{2}\left(Q_{1}\right)} .
$$

Actually if $d\left(Q_{1}, Q_{2}\right) \geq 2 r$, this is (3.2) and if $d\left(Q_{1}, Q_{2}\right) \leq 2 r$, then we use the $L^{2}$-boundedness of $T^{*}$ and $\left(s L^{*}\right)^{\kappa} e^{-s L^{*}}$.

From (3.6), we deduce by duality that

$$
\left\|T(s L)^{\kappa} e^{-s L}(f)\right\|_{L^{2}\left(Q_{2}\right)} \lesssim\left(1+\frac{d\left(Q_{1}, Q_{2}\right)}{r}\right)^{-d-2 N-\delta}\|f\|_{L^{2}\left(Q_{1}\right)} .
$$

To deduce (3.4) when $d\left(Q_{1}, Q_{2}\right) \geq 2 r$, we differentiate the semigroup as follows:

$$
\begin{aligned}
\left(\frac{1}{\mu\left(Q_{2}\right)}\right. & \left.\int_{Q_{2}}\left|T\left(1-e^{-s L}\right)^{\kappa}(f)\right|^{2} d \mu\right)^{1 / 2} \\
& \lesssim\left(\frac{1}{\mu\left(Q_{2}\right)} \int_{Q_{2}}\left|\int_{0}^{s} \cdots \int_{0}^{s} T L^{\kappa} e^{-\left(u_{1}+\cdots+u_{\kappa}\right) L}(f) d u_{1} \cdots d u_{\kappa}\right|^{2} d \mu\right)^{1 / 2} .
\end{aligned}
$$

For each $u_{i} \in(0, s]$, we have to consider $\left(B_{k}\right)_{k}$ and $\left(B_{l}\right)_{l}$, bounded coverings of $Q_{2}$ and $Q_{1}$ by balls of radius $r_{u}=\left(u_{1}+\cdots+u_{\kappa}\right)^{1 / m}$ and then apply (3.7):

$$
\begin{aligned}
\int_{Q_{2}} \mid r_{u}^{m \kappa} & \left.T L^{\kappa} e^{-\left(u_{1}+\cdots+u_{\kappa}\right) L}(f)\right|^{2} d \mu \leq \sum_{k} \int_{B_{k}}\left|r_{u}^{m \kappa} T L^{\kappa} e^{-\left(u_{1}+\cdots+u_{\kappa}\right) L}(f)\right|^{2} d \mu \\
& \lesssim \sum_{k}\left(\sum_{l}\left(1+\frac{d\left(B_{k}, B_{l}\right)}{r_{u}}\right)^{-d-2 N-\delta}\left(\frac{\mu\left(B_{k}\right)}{\mu\left(B_{l}\right)}\right)^{1 / 2}\left(\int_{B_{l}}|f|^{2} d \mu\right)^{1 / 2}\right)^{2} \\
& \lesssim \sum_{k}\left(\sum_{l}\left(1+\frac{d\left(B_{k}, B_{l}\right)}{r_{u}}\right)^{-d-N-\delta}\left(\int_{B_{l}}|f|^{2} d \mu\right)^{1 / 2}\right)^{2} \\
& \lesssim\left(\int_{Q_{1}}|f|^{2} d \mu\right) \sum_{k, l}\left(1+\frac{d\left(B_{k}, B_{l}\right)}{r_{u}}\right)^{-2 d-2 N-2 \delta},
\end{aligned}
$$


where we have used the doubling property and (2.2). Then we note that $d\left(B_{k}, B_{l}\right) \geq$ $d\left(Q_{1}, Q_{2}\right)$ and due to the doubling property,

$$
\begin{aligned}
\sum_{k} 1 & \lesssim\left(\frac{r}{r_{u}}\right)^{d} \sum_{k}\left(\frac{r}{r_{u}}\right)^{-d} \lesssim\left(\frac{r}{r_{u}}\right)^{d} \sum_{k} \frac{\mu\left(B_{k}\right)}{\mu\left(\frac{r}{r_{u}} B_{k}\right)} \\
& \lesssim\left(\frac{r}{r_{u}}\right)^{d} \sum_{k} \frac{\mu\left(B_{k}\right)}{\mu\left(Q_{2}\right)} \\
& \lesssim\left(\frac{r}{r_{u}}\right)^{d} .
\end{aligned}
$$

So it follows with a similar reasoning for the sum over $l$ that

$$
\begin{aligned}
\int_{Q_{2}}\left|r_{u}^{m \kappa} T L^{\kappa} e^{-\left(u_{1}+\cdots+u_{\kappa}\right) L}(f)\right|^{2} d \mu \\
\quad \lesssim\left(\int_{Q_{1}}|f|^{2} d \mu\right)\left(1+\frac{d\left(Q_{1}, Q_{2}\right)}{r_{u}}\right)^{-2 d-2 N-2 \delta}\left(\frac{r}{r_{u}}\right)^{2 d} .
\end{aligned}
$$

We conclude that

$$
\begin{aligned}
& \left(\frac{1}{\mu\left(Q_{2}\right)} \int_{Q_{2}}\left|r_{u}^{m \kappa} T L^{\kappa} e^{-\left(u_{1}+\cdots+u_{\kappa}\right) L}(f)\right|^{2} d \mu\right)^{1 / 2} \\
& \quad \lesssim\left(1+\frac{d\left(Q_{1}, Q_{2}\right)}{r_{u}}\right)^{-d-N-\delta}\left(\frac{r}{r_{u}}\right)^{d}\left(\frac{\mu\left(Q_{1}\right)}{\mu\left(Q_{2}\right)}\right)^{1 / 2}\left(\frac{1}{\mu\left(Q_{1}\right)} \int_{Q_{1}}|f|^{2} d \mu\right)^{1 / 2} .
\end{aligned}
$$

Hence, for $d\left(Q_{1}, Q_{2}\right) \geq 2 r$,

$$
\begin{aligned}
&\left(\frac{1}{\mu\left(Q_{2}\right)}\right.\left.\int_{Q_{2}}\left|T\left(1-e^{-s L}\right)(f)\right|^{2} d \mu\right)^{1 / 2} \\
& \lesssim\left(\frac{\mu\left(Q_{1}\right)}{\mu\left(Q_{2}\right)}\right)^{1 / 2}\left(\frac{1}{\mu\left(Q_{1}\right)} \int_{Q_{1}}|f|^{2} d \mu\right)^{1 / 2} \\
& \times \int_{0}^{\kappa s}\left(1+\frac{d\left(Q_{1}, Q_{2}\right)}{u^{1 / m}}\right)^{-d-N-\delta}\left(\frac{r}{u^{1 / m}}\right)^{d} \frac{d u}{u} \\
& \lesssim\left(\frac{\mu\left(Q_{1}\right)}{\mu\left(Q_{2}\right)}\right)^{1 / 2}\left(1+\frac{d\left(Q_{1}, Q_{2}\right)}{r}\right)^{-d-N-\delta}\left(\frac{1}{\mu\left(Q_{1}\right)} \int_{Q_{1}}|f|^{2} d \mu\right)^{1 / 2} \\
& \lesssim\left(1+\frac{d\left(Q_{1}, Q_{2}\right)}{r}\right)^{-d-\delta}\left(\frac{1}{\mu\left(Q_{1}\right)} \int_{Q_{1}}|f|^{2} d \mu\right)^{1 / 2} .
\end{aligned}
$$

We have used at the first line that

$$
\left|\left\{\left(u_{1}, \cdots, u_{\kappa}\right), u_{1}+\cdots+u_{\kappa}=u\right\}\right| \lesssim u^{\kappa-1} .
$$

If $d\left(Q_{1}, Q_{2}\right) \leq 2 r,(3.10)$ still holds; indeed, we do not have to differentiate and just invoke the $L^{2}$-boundedness of the different appearing operators.

So we have proved that for all balls $Q_{1}, Q_{2}$ of radius $r$, (3.10) holds. Now we deduce (3.4) as follows: we consider $Q$ to be a ball of radius $r$ and $\left(\tilde{Q}_{k}\right)_{k}$ a bounded 
covering of $2^{i} Q \backslash 2^{i-1} Q$ by balls of radius $r$ and then apply (3.10):

$$
\begin{aligned}
&\left(\frac{1}{\mu\left(2^{i} Q\right)} \int_{2^{i} Q \backslash 2^{i-1} Q_{1}}\left|T\left(1-e^{-s L}\right)(f)\right|^{2} d \mu\right)^{1 / 2} \\
& \leq\left(\frac{1}{\mu\left(2^{i} Q\right)} \sum_{k} \int_{\tilde{Q}_{k}}\left|T\left(1-e^{-s L}\right)(f)\right|^{2} d \mu\right)^{1 / 2} \\
& \lesssim\left(\sum_{k}\left(1+\frac{d\left(\tilde{Q}_{k}, Q\right)}{r}\right)^{-2 d-2 \delta} \frac{\mu\left(\tilde{Q}_{k}\right)}{\mu\left(2^{i} Q\right) \mu(Q)} \int_{Q}|f|^{2} d \mu\right)^{1 / 2} \\
& \lesssim 2^{-(d+\delta) i}\left(\frac{1}{\mu(Q)} \int_{Q}|f|^{2} d \mu\right)^{1 / 2} .
\end{aligned}
$$

We have also obtained (3.4) with coefficients $\gamma(j)$ satisfying

$$
\gamma(i) \lesssim 2^{-(d+\delta) i}
$$

Then Theorem 4.2 of [10 yields that $T$ is bounded from the finite atomic Hardy space $H_{L}^{1}$.

Step 3. Interpolation between $H_{L}^{1}$ and $L^{2}$. To obtain interpolation results, we apply Theorem 5.3 of [10]. Aiming at that, off-diagonal estimates of the heat kernel (2.4) imply that

$$
\begin{aligned}
M_{\infty}(f)(x) & :=\sup _{Q \text { ball }}\left\|A_{Q}^{*}(f)\right\|_{L^{\infty}(Q)} \\
& \lesssim \sup _{r>0} \int \frac{1}{\mu(B(y, r))}\left(1+\frac{d(x, y)}{r}\right)^{-d-2 N-\delta}|f(y)| d \mu(y) \\
& \lesssim \sup _{r>0} \frac{1}{\mu(B(x, r))} \int\left(1+\frac{d(x, y)}{r}\right)^{-d-N-\delta}|f(y)| d \mu(y) \\
& \lesssim \mathcal{M}(f)(x),
\end{aligned}
$$

where we have used (2.2) and the fact that $A_{Q}^{*}$ can be expanded as a finite sum of semigroups (with a scale equivalent to $r$, the radius of the ball $Q$ ).

Consequently, we know from Theorem 5.3 [10 that we can interpolate the Hardy space $H_{L}^{1}$ associated to the operators $A_{Q}$ with $L^{2}$ and regain the intermediate Lebesgue spaces as intermediate spaces (see [11 for more details concerning a real interpolation result). This concludes the proof of the theorem for $p \in(1,2]$. We get the result for $p \in(2, \infty)$ by duality, since $T$ and $T^{*}$ satisfy the same assumptions.

In addition, we can obtain weighted estimates. Let us recall the definition of Muckenhoupt's weights and Reverse Hölder classes:

Definition 3.7. A non-negative function $\omega$ on $M$ belongs to the class $\mathbb{A}_{p}$ for $p \in(1, \infty)$ if

$$
\sup _{Q \text { ball }}\left(\frac{1}{\mu(Q)} \int_{Q} w d \mu\right)\left(\frac{1}{\mu(Q)} \int_{Q} \omega^{-1 /(p-1)} d \mu\right)^{p-1}<\infty .
$$


A non-negative function $\omega$ on $M$ belongs to the class $R H_{q}$ for $q \in[1, \infty)$ if there is a constant $C$ such that for every ball $Q \subset X$,

$$
\left(\frac{1}{\mu(Q)} \int_{Q} \omega^{q} d \mu\right)^{1 / q} \leq C\left(\frac{1}{\mu(Q)} \int_{Q} \omega d \mu\right) .
$$

Observe that $R H_{1}$ is the class of all the weights.

For $\omega$ a weight and $p \in[1, \infty]$ an exponent, we write $L^{p}(\omega)$ for the weighted Lebesgue space (associated to the measure $\omega d \mu$ ).

Following the previous Corollary [3.6] and Theorem 6.4 of [10], we get the next result.

Corollary 3.8. Under the assumptions of Theorem 3.3, for all exponents $p \in(1,2)$, let $\omega$ be a weight so that $\omega \in \mathbb{A}_{p} \cap R H_{\left(\frac{2}{p}\right)^{\prime}}$. There is a constant $c_{p}$ such that

$$
\forall f \in \mathcal{S}(M), \quad\left\|T^{*}(f)\right\|_{L^{p}(\omega)}+\|T(f)\|_{L^{p}(\omega)} \leq c_{p}\|f\|_{L^{p}(\omega)} .
$$

For all exponents $p \in(2, \infty)$, let $\omega$ be a weight so that $\omega \in \mathbb{A}_{p / 2}$. There is a constant $c_{p}$ such that

$$
\forall f \in \mathcal{S}(M), \quad\left\|T^{*}(f)\right\|_{L^{p}(\omega)}+\|T(f)\|_{L^{p}(\omega)} \leq c_{p}\|f\|_{L^{p}(\omega)} .
$$

Concerning the condition $\omega \in \mathbb{A}_{p / 2}=\mathbb{A}_{p / 2} \cap R H_{1}$, we recall (Lemma 4.4 of [7]) that for $p \in(2, \infty)$,

$$
\omega \in \mathbb{A}_{p / 2} \cap R H_{1} \Longleftrightarrow \omega^{1-p^{\prime}} \in \mathbb{A}_{p^{\prime}} \cap R H_{\left(\frac{2}{p^{\prime}}\right)^{\prime}}
$$

and the duality for the weighted Lebesgue spaces goes as follows: for $T$ an operator, we recall the fact that $T^{*}$ is the adjoint of $T$ related to the measure $\mu$; so for $p \in(1, \infty)$,

$$
T \text { is } L^{p}(\omega) \text {-bounded } \Longleftrightarrow T^{*} \text { is } L^{p^{\prime}}\left(\omega^{1-p^{\prime}}\right) \text {-bounded. }
$$

3.2. Proof of Theorem 3.3, We devote this subsection to the proof of our main result, Theorem 3.3 Aiming at that, let us define a function: let $\phi$ be the following function defined on $\mathbb{R}^{+}$by

$$
\phi(x):=-\int_{x}^{\infty} t^{\kappa} e^{-t}\left(1-e^{-t}\right)^{2} e^{-t} d t .
$$

Then $\phi$ is a finite sum of polynomial terms, multiplied by decreasing exponentials. So it can be extended in $\mathbb{C}$ and becomes a bounded and holomorphic function in any sector $S_{\omega}$ with $\omega<\pi / 2$ and we have

$$
\phi^{\prime}(x)=x^{\kappa} e^{-x}\left(1-e^{-x}\right)^{2} e^{-x} .
$$

Lemma 3.9. For $f \in L^{2}(X)$, we have the following strong convergence in $L^{2}$ :

- $e^{-t L}(f) \underset{t \rightarrow 0}{\longrightarrow} f$,

- for an integer $k \geq 1,(t L)^{k} e^{-t L}(f) \underset{t \rightarrow 0}{\longrightarrow} 0$,

- for an integer $k,(t L)^{k} e^{-t L}(f) \underset{t \rightarrow \infty}{\longrightarrow} 0$.

Proof. The first two points are due to the $L^{2}$-continuity of the semigroup. For the third point with $k=0$, we remark that the pointwise bound (2.4) yields

$$
\sup _{t>1}\left|e^{-t L}(f)\right| \lesssim \mathcal{M}(f) \in L^{2}
$$


since for every $x \in M$ and $t \geq 1$,

$$
\begin{aligned}
\left|e^{-t L}(f)(x)\right| & \leq \frac{1}{\mu\left(B\left(x, t^{1 / m}\right)\right)} \int\left(1+\frac{d(x, y)}{t^{1 / m}}\right)^{-n-2 N-\delta}|f(y)| d \mu(y) \\
& \lesssim \frac{1}{\mu\left(B\left(x, t^{1 / m}\right)\right)^{1 / 2}}\|f\|_{L^{2}} \underset{t \rightarrow \infty}{\longrightarrow} 0
\end{aligned}
$$

At the second inequality, we have used the homogeneous type of the manifold $M$ and then at the last inequality the infinite measure of $M$. As a consequence, we get that $e^{-t L}(f)$ strongly converges to 0 in $L^{2}$ when $t \rightarrow \infty$. Then, for $k \geq 1$, the limit follows from the uniform $L^{2}$-boundedness of $(t L)^{k} e^{-t L}$.

Proposition 3.10. We have a Sobolev inequality, relative to L: for a large enough integer $M$, for all $t>0$ and a ball $Q$ of radius $r=t^{1 / m}$,

$$
\|f\|_{L^{\infty}(Q)} \lesssim \inf _{Q} \mathcal{M}_{2}\left[(1+t L)^{M} f\right] .
$$

More precisely, for some $\delta>1$ (indeed $\delta$ is the one introduced in (2.4))

$$
\|f\|_{L^{\infty}(Q)} \lesssim \sum_{i \geq 0} 2^{-i \delta}\left(\frac{1}{\mu\left(2^{i} Q\right)} \int_{2^{i} Q}\left|(1+t L)^{M} f\right|^{2} d \mu\right)^{1 / 2}
$$

Proof. It suffices to prove that

$$
\left\|(1+t L)^{-M} f\right\|_{L^{\infty}(Q)} \lesssim \inf _{Q} \mathcal{M}_{2}(f)
$$

(and similarly for the second estimate), which will be provided as soon as we prove the pointwise inequality

$$
\left|r_{t}(x, y)\right| \leq \frac{1}{\mu(B(x, r))}\left(1+\frac{d(x, y)}{r}\right)^{-d-2 N-\delta},
$$

where $r_{t}$ is the kernel of the resolvant $(1+t L)^{-M}$ and $N, d$ are introduced in (2.1) and (2.2).

Aiming at that, we decompose the resolvant with the semigroup as follows (up to a numerical constant):

$$
(1+t L)^{-M}=\int_{0}^{\infty} s^{M} e^{-s(1+t L)} \frac{d s}{s} .
$$

Using the estimates of the heat kernel (2.4), we obtain

$$
\left|r_{t}(x, y)\right| \lesssim \int_{0}^{\infty} s^{M} e^{-s} \frac{1}{\mu\left(B\left(x, r s^{1 / m}\right)\right)}\left(1+\frac{d(x, y)}{r s^{1 / m}}\right)^{-d-2 N-\delta} \frac{d s}{s} .
$$

The integral for $s \in(0,1]$ is bounded by

$$
\begin{aligned}
\int_{0}^{1} s^{M} e^{-s} \frac{1}{\mu\left(B\left(x, r s^{1 / m}\right)\right)} & \left(1+\frac{d(x, y)}{r s^{1 / m}}\right)^{-d-2 N-\delta} \frac{d s}{s} \\
& \lesssim \int_{0}^{1} s^{M} \frac{1}{\mu\left(B\left(x, r s^{1 / m}\right)\right)}\left(1+\frac{d(x, y)}{r}\right)^{-d-2 N-\delta} \frac{d s}{s} \\
& \lesssim\left(1+\frac{d(x, y)}{r}\right)^{-d-2 N-\delta} \int_{0}^{1} s^{M-1} \frac{1}{\mu\left(B\left(x, r s^{1 / m}\right)\right)} d s .
\end{aligned}
$$


Using the doubling property of the measure, it follows that

$$
\mu(B(x, r)) \lesssim \mu\left(B\left(x, r s^{1 / m}\right)\right) s^{-d / m} .
$$

So for $M$ large enough, we have

$$
\begin{aligned}
\int_{0}^{1} s^{M} e^{-s} \frac{1}{\mu\left(B\left(x, r s^{1 / m}\right)\right)} & \left(1+\frac{d(x, y)}{r s^{1 / m}}\right)^{-d-2 N-\delta} \frac{d s}{s} \\
& \lesssim\left(1+\frac{d(x, y)}{r}\right)^{-d-2 N-\delta} \frac{1}{\mu(B(x, r))}
\end{aligned}
$$

which yields the desired inequality for this first term.

Concerning the second part, with $M>(d+2 N+\delta) / m$, we have

$$
\begin{aligned}
& \int_{1}^{\infty} s^{M} e^{-s} \frac{1}{\mu\left(B\left(x, r s^{1 / m}\right)\right)}\left(1+\frac{d(x, y)}{r s^{1 / m}}\right)^{-d-2 N-\delta} \frac{d s}{s} \\
& \qquad \int_{1}^{\infty} s^{-M} \frac{1}{\mu\left(B\left(x, r s^{1 / m}\right)\right)}\left(1+\frac{d(x, y)}{r s^{1 / m}}\right)^{-d-2 N-\delta} \frac{d s}{s} \\
& \lesssim \frac{1}{\mu(B(x, r))} \int_{1}^{\infty} s^{-M}\left(1+\frac{d(x, y)}{r s^{1 / m}}\right)^{-d-2 N-\delta} \frac{d s}{s} \\
& \lesssim \frac{1}{\mu(B(x, r))}\left(1+\frac{d(x, y)}{r}\right)^{-d-2 N-\delta},
\end{aligned}
$$

since for every $v>0$,

$$
\int_{1}^{\infty} s^{-M}\left(1+\frac{v}{s^{1 / m}}\right)^{-d-2 N-\delta} \frac{d s}{s} \simeq(1+v)^{-d-2 N-\delta},
$$

by dividing the integral for $s \leq v^{m}$ and $s \geq v^{m}$. This concludes the proof of (3.13). Then we have

$$
\begin{aligned}
\left\|(1+t L)^{-M} f\right\|_{L^{\infty}(Q)} & \leq \sup _{x \in Q} \int\left|r_{t}(x, y)\right||f(y)| d \mu(y) \\
& \leq \sup _{x \in Q} \frac{1}{\mu(B(x, r))} \int\left(1+\frac{|x-y|}{r}\right)^{-d-2 N-\delta}|f(y)| d \mu(y) \\
& \lesssim \sup _{x \in Q} \frac{1}{\mu(Q)} \int\left(1+\frac{d(y, Q)}{r}\right)^{-d-2 N-\delta}|f(y)| d \mu(y) \\
& \lesssim \sum_{i \geq 0} \frac{1}{\mu(Q)} 2^{-(d+2 N+\delta) i} \mu\left(2^{i} Q\right)^{1 / 2}\|f\|_{L^{2}\left(2^{i} Q\right)} \\
& \lesssim \sum_{i \geq 0} 2^{-\delta i}\left(\inf _{Q} \mathcal{M}_{2}(f)\right)
\end{aligned}
$$

where we have used the doubling property (2.1). This completes the proof of the proposition.

Proof of the first part of Theorem 3.3. The proof is quite long, so we divide it into two steps. First as we use duality, we make precise that the assumptions of Subsection 2.3 assumed for $L$ still hold for the adjoint $L^{*}$ (as they are invariant by duality). 
First step. Reduction to well-localized operators.

Let $f \in L^{2}$ be compactly supported. Then in the weak $L^{2}$-sense we have

$$
\phi(0)^{2} T(f)=\lim _{s \rightarrow 0} \phi(s L) T \phi(s L)(f)
$$

and

$$
0=\lim _{s \rightarrow \infty} \phi(s L) T \phi(s L)(f),
$$

thanks to Lemma 3.9. We can also write

$$
T(f)=-\lim _{\substack{\epsilon \rightarrow 0 \\ R \rightarrow \infty}}[\phi(s L) T \phi(s L)(f)]_{\epsilon}^{R},
$$

which gives

$$
T(f)=-\lim _{\substack{\epsilon \rightarrow 0 \\ R \rightarrow \infty}} \int_{\epsilon}^{R}\left(\left[s \frac{d}{d s} \phi(s L)\right] T \phi(s L)(f)+\phi(s L) T\left[s \frac{d}{d s} \phi(s L)\right] f\right) \frac{d s}{s} .
$$

Given the definition of $\phi$, we have

$$
s \frac{d}{d s} \phi(s L)=(s L)^{\kappa} e^{-s L}\left(1-e^{-s L}\right)^{2} s L e^{-s L}
$$

in order that

$$
\begin{array}{r}
T(f)=-\lim _{\substack{\epsilon \rightarrow 0 \\
R \rightarrow \infty}} \int_{\epsilon}^{R}\left[(s L)^{\kappa} e^{-s L}\left(1-e^{-s L}\right)^{2} s L e^{-s L} T \phi(s L)(f)\right. \\
\left.+\phi(s L) T(s L)^{\kappa} e^{-s L}\left(1-e^{-s L}\right)^{2} s L e^{-s L} f\right] \frac{d s}{s} .
\end{array}
$$

The second term can be seen as the adjoint of the first term by replacing $L$ with $L^{*}$ (and $T$ with $T^{*}$ ). As our assumptions are invariant by duality, it is also sufficient to prove a uniform bound with respect to $\epsilon$ and $R$ of $\left\|U_{\epsilon, R}(f)\right\|_{L^{2}}$ with

$$
U_{\epsilon, R}(f):=\int_{\epsilon}^{R}\left(1-e^{-s L}\right) s L e^{-s L} T_{s} \phi(s L)(f) \frac{d s}{s},
$$

where we set $T_{s}:=(s L)^{\kappa} e^{-s L}\left(1-e^{-s L}\right) T$.

According to the assumptions, it is easy to check that $T_{s}$ satisfies the following two properties:

- The map $(s, x) \rightarrow T_{s^{m}}(\mathbf{1})(x)$ defines a Carleson measure.

This comes from $T_{s}(\mathbf{1})=(s L)^{\kappa} e^{-s L}\left(1-e^{-s L}\right) T(\mathbf{1})$ and Proposition 2.12

- For all $s$, the operator $T_{s}$ has off-diagonal decays at the scale $s$ : for all $L^{2}$-functions $f, g$ supported on the balls $Q_{1}, Q_{2}$ of radius $r:=s^{1 / m}$, we have

$$
\left|\left\langle T_{s}(\phi(s L) f), g\right\rangle\right| \lesssim\left(1+\frac{d\left(Q_{1}, Q_{2}\right)}{r}\right)^{-d-2 N-\delta}\|f\|_{L^{2}\left(Q_{1}\right)}\|g\|_{L^{2}\left(Q_{2}\right)} .
$$

This comes from $\left\langle T_{s}(\phi(s L) f), g\right\rangle=\left\langle(s L)^{\kappa}\left(1-e^{-s L}\right) e^{-s L} T(\phi(s L) f), g\right\rangle$ and assumption (3.1) together with the arguments of Remark 3.1)(if $d\left(Q_{1}, Q_{2}\right) \geq$ $2 r$ ) and assumption (3.3) (if $d\left(Q_{1}, Q_{2}\right) \leq 2 r$ ). 
Second step. Study of $U_{\epsilon, R}$.

We decompose $U_{\epsilon, R}$ as follows:

$$
\begin{aligned}
U_{\epsilon, R}(f)= & \int_{\epsilon}^{R}\left(1-e^{-s L}\right) L e^{-s L}\left[T_{s}(\mathbf{1}) \phi(s L)(f)\right] d s \\
& +\int_{\epsilon}^{R}\left(1-e^{-s L}\right) L e^{-s L}\left[T_{s} \phi(s L)-T_{s}(\mathbf{1}) \phi(s L)\right](f) d s \\
& :=\operatorname{Main}(f)+\operatorname{Error}(f) .
\end{aligned}
$$

The main term is controlled by duality: in pairing with any $L^{2}$-function $g$,

$$
\begin{aligned}
|\langle\operatorname{Main}(f), g\rangle| & =\left|\int_{\epsilon}^{R}\left\langle\left(1-e^{-s L}\right) s L e^{-s L}\left[T_{s}(\mathbf{1}) \phi(s L)(f)\right], g\right\rangle \frac{d s}{s}\right| \\
& =\left|\int_{\epsilon}^{R}\left\langle T_{s}(\mathbf{1}) \phi(s L)(f),\left(1-e^{-s L^{*}}\right) s L^{*} e^{-s L^{*}} g\right\rangle \frac{d s}{s}\right| \\
& \leq\left\|T_{s}(\mathbf{1}) \phi(s L)(f)\right\|_{L^{2}\left(\mathbb{R}^{+} \times M\right)}\left\|\left(1-e^{-s L^{*}}\right) s L^{*} e^{-s L^{*}} g\right\|_{L^{2}\left(\mathbb{R}^{+} \times M\right)},
\end{aligned}
$$

where $\mathbb{R}^{+} \times M$ is equipped with the tensorial-product measure $\frac{d s}{s} \otimes d \mu(x)$. The second quantity is bounded by $\|g\|_{L^{2}}$ by the $L^{2}$-boundedness of the LittlewoodPaley function (see Remark 2.8 applied to $L^{*}$ ). Since $T_{s^{m}}(\mathbf{1})$ is a Carleson measure, it is well known that we get the following inequality, making it appear as a nontangential maximal function

$$
\|\operatorname{Main}(f)\|_{L^{2}} \lesssim\left\|\sup _{\substack{(s, y) \in \mathbb{R}^{+} \times M \\|x-y| \leq s}}\left|\phi\left(s^{m} L\right) f(y)\right|\right\|_{L^{2}} .
$$

This quantity can be estimated by $\|f\|_{L^{2}}$ since the maximal function is pointwisely bounded by $\mathcal{M}(f)$ thanks to the pointwise estimates on the heat kernel (2.4) and Remark 2.8

It also remains to study the "Error term" in (3.15). For this term, we use duality again as follows:

$$
\begin{aligned}
& |\langle\operatorname{Error}(f), g\rangle| \\
& \quad \leq\left|\int_{M} \int_{\epsilon}^{R} s L^{*} e^{-s L^{*}}\left(1-e^{-s L^{*}}\right)(g)(x)\left[T_{s} \phi(s L)-T_{s}(\mathbf{1}) \phi(s L)\right](f)(x) \frac{d s}{s} d \mu(x)\right| .
\end{aligned}
$$

The Cauchy-Schwarz inequality yields

$$
\begin{aligned}
|\langle\operatorname{Error}(f), g\rangle| & \lesssim\left(\int_{\epsilon}^{R} \int_{M}\left|s L^{*} e^{-s L^{*}}\left(1-e^{-s L^{*}}\right)(g)\right|^{2} \frac{d \mu d t}{t}\right)^{1 / 2} \\
& \times\left(\int_{M} \int_{\epsilon}^{R}\left|\left[T_{s} \phi(s L)-T_{s}(\mathbf{1}) \phi(s L)\right](f)\right|^{2} \frac{d s d \mu}{s}\right)^{1 / 2} \\
& \lesssim\|g\|_{L^{2}}\left(\int_{M} \int_{\epsilon}^{R}\left|\left[T_{s} \phi(s L)-T_{s}(\mathbf{1}) \phi(s L)\right](f)\right|^{2} \frac{d s d \mu}{s}\right)^{1 / 2}
\end{aligned}
$$


where we have used the $L^{2}$-boundedness of the square function (see Remark 2.8). Let us fix $s \in(\epsilon, R)$ and consider

$$
\begin{aligned}
I_{s} & :=\int\left|\left[T_{s} \phi(s L)-T_{s}(\mathbf{1}) \phi(s L)\right](f)(x)\right|^{2} d \mu(x) \\
& =\int\left|T_{s}[\phi(s L) f-\phi(s L) f(x)](x)\right|^{2} d \mu(x) .
\end{aligned}
$$

We choose $\left(Q_{i}\right)_{i}$ to be a bounded covering of the whole manifold $M$ with balls of radius $r:=s^{1 / m}$, in order that

$$
\begin{aligned}
I_{s} & \leq \sum_{i} \int_{Q_{i}}\left|T_{s}[\phi(s L) f-\phi(s L) f(x)](x)\right|^{2} d \mu(x) \\
& \leq \sum_{i} \int_{Q_{i}} \sup _{y \in Q_{i}}\left|T_{s}[\phi(s L) f-\phi(s L) f(y)](x)\right|^{2} d \mu(x) .
\end{aligned}
$$

The supremum over $y \in Q_{i}$ can be estimated with the Sobolev inequality, Proposition 3.10. Let us fix the index $i$. For a large enough integer $M$, Proposition 3.10 implies

$$
\begin{aligned}
& \sup _{y \in Q_{i}}\left|T_{s}[\phi(s L) f-\phi(s L) f(y)](x)\right|^{2} \\
& \quad \lesssim \sum_{j \geq 0} 2^{-2 \delta j} \frac{1}{\mu\left(2^{j} Q_{i}\right)} \int_{2^{j} Q_{i}}\left|(1+s L)^{M}\left[T_{s}[\phi(s L) f-\phi(s L) f(\cdot)](x)\right](y)\right|^{2} d \mu(y),
\end{aligned}
$$

with another exponent $\delta$, which is still strictly greater than 1 .

By expanding $(1+s L)^{M}$ and using $L(\mathbf{1})=0$, we have

$$
\begin{array}{r}
(1+s L)^{M}\left[T_{s}[\phi(s L) f-\phi(s L) f(\cdot)](x)\right](y)=T_{s}[\phi(s L) f-\phi(s L) f(y)](x) \\
-T_{s}(\mathbf{1})(x)\left[(1+s L)^{M}-1\right] \phi(s L) f(y) .
\end{array}
$$

Consequently,

$$
\begin{aligned}
\mid T_{s}[\phi(s L) f & -\phi(s L) f(y)]\left.(x)\right|^{2} \\
& \lesssim \sum_{j \geq 0} 2^{-2 \delta j} \frac{1}{\mu\left(2^{j} Q_{i}\right)} \int_{2^{j} Q_{i}}\left|T_{s}[\phi(s L) f-\phi(s L) f(y)](x)\right|^{2} d \mu(y) \\
& +\left|T_{s}(\mathbf{1})(x)\right|^{2} \sum_{j \geq 0} 2^{-2 \delta j} \frac{1}{\mu\left(2^{j} Q_{i}\right)} \int_{2^{j} Q_{i}}\left|\left[(1+s L)^{M}-1\right] \phi(s L) f\right|^{2} d \mu .
\end{aligned}
$$

Finally, we get

$$
\begin{aligned}
& I_{s} \leq \sum_{i} \sum_{j \geq 0} 2^{-2 \delta j} \frac{1}{\mu\left(2^{j} Q_{i}\right)} \int_{Q_{i}} \int_{2^{j} Q_{i}}\left|T_{s}[\phi(s L) f-\phi(s L) f(y)](x)\right|^{2} d \mu(y) d \mu(x) \\
& +\sum_{i} \sum_{j \geq 0} 2^{-2 \delta j} \frac{1}{\mu\left(2^{j} Q_{i}\right)}\left(\int_{Q_{i}}\left|T_{s} \mathbf{1}\right|^{2} d \mu\right)\left(\int_{2^{j} Q_{i}}\left|\left[(1+s L)^{M}-1\right] \phi(s L) f\right|^{2} d \mu\right) .
\end{aligned}
$$

Let us denote by $I_{s}^{1}$ and $I_{s}^{2}$ the two previous terms. 
Concerning $I_{s}^{1}$, we use Fubini's Theorem and the off-diagonal estimates (3.14) and deduce

$$
\begin{gathered}
I_{s}^{1} \leq \sum_{i} \sum_{j \geq 0} 2^{-2 \delta j} \frac{1}{\mu\left(2^{j} Q_{i}\right)} \int_{Q_{i}} \int_{2^{j} Q_{i}}\left|T_{s}[\phi(s L) f-\phi(s L) f(y)](x)\right|^{2} d \mu(y) d \mu(x) \\
\lesssim \sum_{j \geq 0} 2^{-2 \delta j} \sum_{\substack{i, l_{1} \\
Q_{l_{1}} \subset 2^{j} Q_{i}}} \int_{Q_{i}}\left[\sum_{l_{2}}\left(1+\frac{d\left(Q_{l_{1}}, Q_{l_{2}}\right)}{s^{1 / m}}\right)^{-d-2 N-\delta}\right. \\
\left.\times\left(\int_{Q_{l_{2}}}|\phi(s L) f(x)-\phi(s L) f(y)|^{2} \frac{d \mu(y)}{\mu\left(2^{j} Q_{i}\right)}\right)^{1 / 2}\right]^{2} d \mu(x) .
\end{gathered}
$$

To be more precise, from (3.14) we have off-diagonal decays for $T_{s}\left(e^{-s L} \cdot\right)$ at the scale $s$. We can also obtain off-diagonal decays for $T_{s}\left(e^{-s L / 2} \cdot\right)$ at the same scale $s$. So at the last line of the previous inequality, $e^{s L / 2} \phi(s L)$ should appear (and not just $\phi(s L))$. For easy readibility, we prefer to keep the notation $\phi(s L)$ : indeed $\phi(s L)$ is equal to a polynomial term multiplied by $e^{-s L}$; so $\phi(s L)$ and $e^{s L / 2} \phi(s L)$ satisfy the same off-diagonal estimates at the scale $s$.

Since $\delta>1$, with another constant $\delta^{\prime}>1$ (indeed belonging in $(1, \delta)$ ), the Cauchy-Schwarz inequality gives

$$
\begin{aligned}
& {\left[\sum_{l_{2}}\left(1+\frac{d\left(Q_{l_{1}}, Q_{l_{2}}\right)}{s^{1 / m}}\right)^{-d-2 N-\delta}\left(\int_{Q_{l_{2}}}|\phi(s L) f(x)-\phi(s L) f(y)|^{2} \frac{d \mu(y)}{\mu\left(2^{j} Q_{i}\right)}\right)^{1 / 2}\right]^{2}} \\
& \lesssim\left[\sum_{l_{2}}\left(1+\frac{d\left(Q_{l_{1}}, Q_{l_{2}}\right)}{s^{1 / m}}\right)^{-d-2 N-2 \delta^{\prime}} \int_{Q_{l_{2}}}|\phi(s L) f(x)-\phi(s L) f(y)|^{2} \frac{d \mu(y)}{\mu\left(2^{j} Q_{i}\right)}\right],
\end{aligned}
$$

since for each $l_{1}$,

$$
\sum_{l_{2}}\left(1+\frac{d\left(Q_{l_{1}}, Q_{l_{2}}\right)}{s^{1 / m}}\right)^{-d-2\left(\delta-\delta^{\prime}\right)} \lesssim 1 .
$$

Then Proposition 2.7 and the Poincaré inequality $\left(P_{2}\right)$ yield

$$
\begin{aligned}
I_{s}^{1} & \lesssim \sum_{j \geq 0} 2^{-2 \delta^{\prime} j} \sum_{\substack{i, l_{1}, l_{2} \\
Q_{l_{1} \subset 2^{j} Q_{i}}}}\left(1+\frac{d\left(Q_{l_{1}}, Q_{l_{2}}\right)}{s^{1 / m}}\right)^{-d-2 N-2 \delta^{\prime}}\left(d\left(Q_{l_{1}}, Q_{l_{2}}\right)^{2}+2^{2 j} s^{2 / m}\right) \\
& \times\left[\frac{\mu\left(Q_{i}\right)}{\mu\left(2^{j} Q_{i}\right)} \int_{\substack{Q_{l_{2}} \\
\mathcal{M}_{2-\epsilon}}}[\nabla \phi(s L) f]^{2} d \mu+\frac{\mu\left(Q_{l_{2}}\right)}{\mu\left(2^{j} Q_{i}\right)} \int_{Q_{i}} \mathcal{M}_{2-\epsilon}[\nabla \phi(s L) f]^{2} d \mu\right],
\end{aligned}
$$

which can be divided into two quantities (we have used that $d\left(Q_{i}, Q_{l_{2}}\right) \leq d\left(Q_{l_{1}}, Q_{l_{2}}\right)$ $\left.+2^{j} s^{1 / m}\right)$. For the first quantity, we can compute the sum over $i$ since for every $l_{1}$,

$$
\sum_{\substack{i \\ Q_{l_{1}} \subset 2^{j} Q_{i}}} \frac{\mu\left(Q_{i}\right)}{\mu\left(2^{j} Q_{i}\right)} \lesssim \sum_{\substack{i \\ Q_{i} \subset 2^{j} Q_{l_{1}}}} \frac{\mu\left(Q_{i}\right)}{\mu\left(2^{j} Q_{l_{1}}\right)} \lesssim 1
$$


where we have used that $Q_{l_{1}} \subset 2^{j} Q_{i}$ implies $Q_{i} \subset 2^{j} Q_{l_{1}}$ and $2^{j+2} Q_{i} \simeq 2^{j+2} Q_{l_{1}}$. For the second quantity, we can estimate the sum over $l_{2}$ thanks to for every $l_{1}$,

$$
\begin{aligned}
\sum_{l_{2}}\left(1+\frac{d\left(Q_{l_{1}}, Q_{l_{2}}\right)}{s^{1 / m}}\right)^{-d-2 N-2\left(\delta^{\prime}-1\right)} & \mu\left(Q_{l_{2}}\right) \\
& \lesssim \mu\left(Q_{l_{1}}\right) \sum_{l_{2}}\left(1+\frac{d\left(Q_{l_{1}}, Q_{l_{2}}\right)}{s^{1 / m}}\right)^{-d-2\left(\delta^{\prime}-1\right)} \\
& \lesssim \mu\left(Q_{l_{1}}\right) \sum_{k} 2^{-k\left(d+2 \delta^{\prime}-2\right)} \sum_{\substack{l_{2} \\
d\left(Q_{l_{2}}, Q_{l_{1}}\right) 2^{k} s}} 1 \\
& \lesssim \mu\left(Q_{l_{1}}\right) \sum_{k} 2^{-k\left(d+2 \delta^{\prime}-2\right)} 2^{k d} \lesssim \mu\left(Q_{l_{1}}\right)
\end{aligned}
$$

due to the doubling property, $\delta^{\prime}>1$ and arguments similar to (3.8). So we obtain:

$$
\begin{aligned}
& I_{s}^{1} \\
& \lesssim \sum_{j \geq 0} 2^{-2\left(\delta^{\prime}-1\right) j} \sum_{l_{1}, l_{2}}\left(1+\frac{d\left(Q_{l_{1}}, Q_{l_{2}}\right)}{s^{1 / m}}\right)^{-d-2 N-2\left(\delta^{\prime}-1\right)} \int_{Q_{l_{2}}} \mathcal{M}_{2-\epsilon}\left[s^{1 / m} \nabla \phi(s L) f\right]^{2} d \mu \\
& +\sum_{j \geq 0} 2^{-2\left(\delta^{\prime}-1\right) j} \sum_{\substack{i, l_{1} \\
Q_{l_{1}} \subset 2^{j} Q_{i}}} \frac{\mu\left(Q_{l_{1}}\right)}{\mu\left(2^{j} Q_{i}\right)} \int_{Q_{i}} \mathcal{M}_{2-\epsilon}\left[s^{1 / m} \nabla \phi(s L) f\right]^{2} d \mu \\
& \lesssim \sum_{l_{2}} \int_{Q_{l_{2}}} \mathcal{M}_{2-\epsilon}\left[s^{1 / m} \nabla \phi(s L) f\right]^{2} d \mu \\
& +\sum_{j \geq 0} 2^{-2\left(\delta^{\prime}-1\right) j} \sum_{i} \int_{Q_{i}} \mathcal{M}_{2-\epsilon}\left[s^{1 / m} \nabla \phi(s L) f\right]^{2} d \mu \\
& \lesssim \int \mathcal{M}_{2-\epsilon}\left[s^{1 / m} \nabla \phi(s L) f\right]^{2} d \mu \text {. }
\end{aligned}
$$

We have used the bounded-overlap property of $\left(Q_{i}\right)_{i}$ (balls of radius $s$ ). By the $L^{2}$-boundedness of the maximal operator $\mathcal{M}_{2-\epsilon}$ (see Theorem [2.3), we conclude that

$$
I_{s}^{1} \lesssim \int\left|s^{1 / m} \nabla \phi(s L) f\right|^{2} d \mu .
$$

It remains to study the second term $I_{s}^{2}$, which is equal to

$$
\begin{aligned}
I_{s}^{2}=\sum_{i} \sum_{j \geq 0} 2^{-2 \delta j} \frac{1}{\mu\left(2^{j} Q_{i}\right)} & \left(\int_{Q_{i}}\left|T_{s} \mathbf{1}(x)\right|^{2} d \mu(x)\right) \\
\times & \left(\int_{2^{j} Q_{i}}\left|\left[(1+s L)^{M}-1\right] \phi(s L) f(y)\right|^{2} d \mu(y)\right) .
\end{aligned}
$$

Since $T_{s} \mathbf{1}(x)=(s L)^{\kappa} e^{-s L}\left(1-e^{-s L}\right) T(\mathbf{1})$ and $T(\mathbf{1})$ belongs to $B M O_{L}$, Proposition 2.12 yields

$$
\int_{Q_{i}}\left|T_{s} \mathbf{1}(x)\right|^{2} d x \lesssim \mu\left(Q_{i}\right)
$$


Hence,

$$
\begin{aligned}
I_{s}^{2} & \lesssim \sum_{i} \sum_{j \geq 0} 2^{-2 \delta j} \frac{\mu\left(Q_{i}\right)}{\mu\left(2^{j} Q_{i}\right)} \int_{2^{j} Q_{i}}\left|\left[(1+s L)^{M}-1\right] \phi(s L) f(y)\right|^{2} d \mu(y) \\
& \lesssim \int\left|\left[(1+s L)^{M}-1\right] \phi(s L) f\right|^{2} d \mu
\end{aligned}
$$

where we have used similar arguments to (3.8) in order to prove that for each integer $j$, the collection $\left(2^{j} Q_{i}\right)_{i}$ is a $2^{j d}$-bounded covering:

$$
\sum_{i} \mathbf{1}_{2^{j} Q_{i}} \lesssim 2^{j d}
$$

Consequently, from (3.17) and (3.18) we obtain

$$
I_{s} \lesssim \int\left|s^{1 / m} \nabla \phi(s L) f\right|^{2}+\left|\left[(1+s L)^{M}-1\right] \phi(s L) f\right|^{2} d \mu,
$$

which finally yields

$$
\begin{aligned}
& |\langle\operatorname{Error}(f), g\rangle| \lesssim\|g\|_{L^{2}} \\
& \quad \times\left(\int_{\epsilon}^{R} \int\left|s^{1 / m} \nabla \phi(s L) f(y)\right|^{2}+\left|\left[(1+s L)^{M}-1\right] \phi(s L) f(y)\right|^{2} \frac{d \mu(y) d s}{s}\right)^{1 / 2} .
\end{aligned}
$$

We also obtain the desired estimate

$$
\|\operatorname{Error}(f)\|_{L^{2}} \lesssim\|f\|_{L^{2}}
$$

by invoking assumption (2.6) for the first quantity and point 2 of Remark 2.8 for the second one. Since the two terms Main $(f)$ and $\operatorname{Error}(f)$ of (3.15) have been bounded in $L^{2}$, we have also proved that $U_{\epsilon, R}$ is uniformly (with respect to $\epsilon, R$ ) bounded on $L^{2}$. The second step is also finished and the proof of the first part of the theorem too.

Proof of the second part of Theorem 3.3. We assume that $T$ admits a continuous extension from $L^{2}$ to $L^{2}$. By symmetry, it suffices for us to check that $T(\mathbf{1})$ belongs to $B M O_{L}$. By definition of $B M O_{L}$ (see Subsection 2.4), we have to show that

$$
\sup _{\substack{Q \\ r_{Q}=t^{1 / m}}} \frac{1}{\mu(Q)} \int_{Q}\left|T(\mathbf{1})-e^{-t L} T(\mathbf{1})\right| d \mu<\infty .
$$

We recall that $T(\mathbf{1})$ is assumed to be well defined. Indeed, we will prove a weaker but equivalent property

$$
\sup _{Q}\left(\frac{1}{\mu(Q)} \int_{Q}\left|T(\mathbf{1})-e^{-t L} T(\mathbf{1})\right|^{2} d \mu\right)^{1 / 2}<\infty
$$

due to the John-Nirenberg inequality (see Theorem 3.1 in 24 for a proof of such properties in this general framework concerning semigroups and 12] for an extension of such results in a more abstract setting). 
Let us fix a ball $Q$ of radius $r:=t^{1 / m}$ and by duality a function $f \in L^{2}(Q)$ such that $\|f\|_{L^{2}(Q)} \leq \mu(Q)^{-1 / 2}$. We have to bound

$$
\left|\left\langle\left(1-e^{-t L}\right) T(\mathbf{1}), f\right\rangle\right|=\left|\left\langle T(\mathbf{1}),\left(1-e^{-t L^{*}}\right) f\right\rangle\right| .
$$

First we denote by $\chi_{Q}$ the characteristic function of $4 Q$ and, by assumption, we have

$$
\begin{aligned}
\left|\left\langle T\left(\chi_{Q}\right),\left(1-e^{-t L^{*}}\right) f\right\rangle\right| & \lesssim\left\|\chi_{Q}\right\|_{L^{2}}\|f\|_{L^{2}} \\
& \lesssim \mu(Q)^{1 / 2} \mu(Q)^{-1 / 2} \lesssim 1,
\end{aligned}
$$

where we have used the doubling property and the $L^{2}$-boundedness of the semigroup. So we have to bound the remainder term, which we differentiate as follows:

$$
\left|\left\langle T\left(1-\chi_{Q}\right),\left(1-e^{-t L^{*}}\right) f\right\rangle\right| \leq \int_{0}^{t}\left|\left\langle s L e^{-s L} T\left(1-\chi_{Q}\right), f\right\rangle\right| \frac{d s}{s} .
$$

For each $s$ and each integer $j \geq 1$, we consider $\left(Q_{l}^{s, j}\right)_{l}$ to be a bounded covering of $2^{j} Q \backslash 2^{j-1} Q$ by balls of radius $s^{1 / m}$ and we associate $\chi_{l}^{s, j}$ with a partition of unity, in order that

$$
\chi_{Q}+\sum_{j \geq 1} \sum_{l} \chi_{l}^{s, j}=\mathbf{1}
$$

Similarly, let $\left(Q_{l}^{s}\right)_{l}$ be a bounded covering of $Q$ by balls of radius $s^{1 / m}$ and associate $\chi_{l}^{s}$ with a partition of unity, in order that

$$
f=\sum_{l} f \chi_{l}^{s} .
$$

From the off-diagonal decay (3.1) and since $\kappa=1$, this yields

$$
\begin{aligned}
& \left|\left\langle s L e^{-s L} T\left(1-\chi_{Q}\right), f\right\rangle\right| \lesssim \sum_{j \geq 0} \sum_{l_{1}, l_{2}}\left|\left\langle s L e^{-s L} T\left(\chi_{l_{1}}^{j, s}\right), f \chi_{l_{2}}^{s}\right\rangle\right| \\
& \lesssim \sum_{j \geq 1} \sum_{l_{1}, l_{2}}\left\|\chi_{l_{1}}^{j, s}\right\|_{L^{2}}\left\|f \chi_{l_{2}}^{s}\right\|_{L^{2}}\left(1+\frac{2^{j} r}{s^{1 / m}}\right)^{-d-\delta} \\
& \lesssim \sum_{j \geq 1}\left(\sum_{l_{1}} \mu\left(Q_{l_{1}}^{j, s}\right)\right)^{1 / 2}\left(\sum_{l_{2}}\left\|f \chi_{l_{2}}^{s}\right\|_{L^{2}}^{2}\right)^{1 / 2}\left(\frac{2^{j} r}{s^{1 / m}}\right)^{d / 2}\left(\frac{r}{s^{1 / m}}\right)^{d / 2}\left(\frac{s^{1 / m}}{2^{j} r}\right)^{d+\delta} \\
& \lesssim \sum_{j \geq 1} \mu\left(2^{j} Q\right)^{1 / 2} \mu(Q)^{-1 / 2}\left(\frac{r}{s^{1 / m}}\right)^{-\delta} 2^{-j(d / 2+\delta)} \\
& \lesssim \sum_{j \geq 1} 2^{-j \delta}\left(\frac{s^{1 / m}}{r}\right)^{\delta} \\
& \lesssim\left(\frac{s^{1 / m}}{r}\right)^{\delta} .
\end{aligned}
$$

We have used the Cauchy-Schwarz inequality in $l_{1}$ and $l_{2}$ with as previously (see arguments employed for (3.80)

$$
\sum_{l_{1}} 1 \lesssim\left(\frac{2^{j} r}{s^{1 / m}}\right)^{d} \quad \text { and } \quad \sum_{l_{2}} 1 \lesssim\left(\frac{r}{s^{1 / m}}\right)^{d}
$$


By integrating for $s \in(0, t)$, we deduce

$$
\left|\left\langle T\left(1-\chi_{Q}\right),\left(1-e^{-t L^{*}}\right) f\right\rangle\right| \leq \int_{0}^{t}\left(\frac{s^{1 / m}}{r}\right)^{\delta} \frac{d s}{s} \lesssim 1,
$$

which concludes the proof of (3.19).

\section{Applications to new paraproducts and to Calderón-Zygmund OPERATORS ON A RIEMANNIAN MANIFOLD}

We consider an operator $L$ satisfying the assumptions of the previous sections. Moreover we will assume off-diagonal decays for the gradient of the semigroup as follows: for all $s>0$ and all balls $Q_{1}, Q_{2}$ of radius $r=s^{1 / m}$, we have for every integer $k \geq 0$,

$$
\left\|s^{1 / m} \nabla(s L)^{k} e^{-s L}(f)\right\|_{L^{2}\left(Q_{2}\right)} \lesssim\left(1+\frac{d\left(Q_{1}, Q_{2}\right)}{r}\right)^{-d-2 N-\delta}\|f\|_{L^{2}\left(Q_{1}\right)} .
$$

We just emphasize that this new assumption still holds in the examples described in Subsection 2.5. Indeed, usually, the gradient of the semigroup satisfies the Gaffney estimates.

We want to study new kinds of paraproducts, relying on the semigroup. For $b \in L^{\infty} \subset B M O_{L}$ (indeed by Remark 7.6 in [10] and Proposition 6.7 in [23, we know that $L^{\infty} \subset B M O \subset B M O_{L}$ thanks to $\left.L(\mathbf{1})=0\right)$ and $f, g \in L^{2}$, let us consider the trilinear form

$$
\Lambda^{1}(b, f, g):=\int_{M} \int_{0}^{\infty}\left[\psi_{t}\left(L^{*}\right) g\right]\left[\phi_{t}(L) b \psi_{t}(L) f\right] \frac{d t}{t} d \mu,
$$

where we write for convenience

$$
\psi_{t}(L):=(t L)^{N} e^{-t L}\left(1-e^{-t L}\right) \quad \text { and } \quad \phi_{t}(L):=e^{-t L},
$$

with a large enough integer $N>d / m$.

As a direct consequence of the Cauchy-Schwarz inequality, the pointwise bound on $\phi_{t}(L)$ and quadratic estimates (due to Remark 2.8), we know that $\Lambda^{1}$ is bounded on $L^{\infty} \times L^{2} \times L^{2}$. Then we deduce the following result.

Proposition 4.1. The trilinear form $\Lambda^{1}$ is bounded on $L^{\infty} \times L^{p} \times L^{p^{\prime}}$ for every exponent $p \in(1, \infty)$.

Proof. Let us fix the function $b \in L^{\infty}$ and consider the linear operator $U$ such that

$$
\langle U(f), g\rangle:=\Lambda^{1}(b, f, g) .
$$

It is given by

$$
U(f):=\int_{0}^{\infty} \psi_{t}(L)\left[\phi_{t}(L) b \psi_{t}(L) f\right] \frac{d t}{t} .
$$

We have just seen that $T$ is bounded on $L^{2}$. Then, we leave the details to the reader and refer to Proposition 4.5 (where we prove a stronger result). It is quite easy to check that $U$ satisfies assumptions (3.1), (3.2) and (3.3). We also deduce the desired result by applying Corollary 3.6 . 
We are now looking to invert the role of the $L^{\infty}$-function $b$ and the $L^{2}$-function $f$ :

Proposition 4.2. The trilinear form $\Lambda^{2}$, defined by

$$
\Lambda^{2}(b, f, g):=\int_{M} \int_{0}^{\infty}\left[\psi_{t}\left(L^{*}\right) g\right]\left[\phi_{t}(L) f \psi_{t}(L) b\right] \frac{d t}{t} d \mu,
$$

is bounded on $L^{\infty} \times L^{p} \times L^{p^{\prime}}$ for every exponent $p \in(1, \infty)$.

Proof. Using the Carleson measure property (Proposition 2.12 and since $L^{\infty} \subset$ $\left.B M O_{L}\right)$ together with the Cauchy-Schwarz inequality, we obtain the desired result for $p=2$. By the same reasoning as used for Proposition 4.1, we conclude this proof.

Using trilinear interpolation and symmetry, we deduce the following result.

Proposition 4.3. The trilinear form $\Lambda$ defined by

$$
\Lambda(h, f, g):=\int_{0}^{\infty} \int_{M} \psi_{t}(L) g \phi_{t}(L) f \psi_{t}(L) h \frac{d t}{t} d \mu,
$$

is bounded on $L^{p} \times L^{q} \times L^{r}$ for all exponents $p, q, r \in(1, \infty]$ satisfying

$$
\frac{1}{p}+\frac{1}{q}+\frac{1}{r}=1
$$

These results concerning paraproducts with two functions $\psi_{t}$ are also easily obtained, thanks to duality and the Cauchy-Schwarz inequality in the variable $t$. Let us note that we do not need $N>d / m$. We are now interested in paraproducts, involving only one function $\psi_{t}$.

Remark 4.4. Let us first examine this situation in the "classical case". Let us consider the Euclidean space $M=\mathbb{R}^{d}$ and denote by $\Psi$ a smooth function on $\mathbb{R}^{d}$ whose spectrum is contained in a corona around 0 and by $\Phi$ another smooth function with a bounded spectrum. Then with the usual notation $\Psi_{t}:=t^{-d} \Psi(. / t)$ and similarly for $\Phi$, we are interested in the following paraproducts:

$$
f \rightarrow \int_{0}^{\infty} \Psi_{t}\left[\Phi_{t}(f) \Phi_{t}(b)\right] \frac{d t}{t},
$$

with $b \in L^{\infty}$. By duality, this gives rise to the following trilinear function:

$$
(b, f, g) \rightarrow \int_{0}^{\infty} \int_{\mathbb{R}^{d}} \Psi_{t}(g) \Phi_{t}(f) \Phi_{t}(b) \frac{d t d x}{t} .
$$

Since we know that the spectrum of a product is contained in the Minkowski sum of the spectrums, it follows that we can find some "good" smooth functions $\tilde{\Psi}$ and $\tilde{\Phi}$ (satisfying the same spectral property as $\Psi$ and $\Phi$ ) such that

$$
\begin{aligned}
\int_{\mathbb{R}^{d}} \int_{0}^{\infty} \Psi_{t}(g) \Phi_{t}(b) \Phi_{t}(b) \frac{d t d x}{t}= & \int_{\mathbb{R}^{d}} \int_{0}^{\infty} \Psi_{t}(g) \tilde{\Phi}_{t}(f) \tilde{\Psi}_{t}(b) \frac{d t d x}{t} \\
& +\int_{\mathbb{R}^{d}} \int_{0}^{\infty} \Psi_{t}(g) \tilde{\Psi}_{t}(f) \tilde{\Phi}_{t}(b) \frac{d t d x}{t} .
\end{aligned}
$$

So it happens that such paraproducts can be reduced to the sum of two paraproducts involving "two functions $\Psi$ ". Hence, they are bounded in Lebesgue spaces.

This reduction is due to the frequency analysis of the product. It is not clear how we can apply a similar reasoning in the framework of semigroups. This is the goal of the two following subsections, via our $T(1)$-theorem. 


\subsection{Boundedness of new paraproducts in Lebesgue spaces with $r^{\prime}>1$.}

Theorem 4.5. The trilinear form $\Lambda$, defined by

$$
\Lambda(h, f, g):=\int_{M} \int_{0}^{\infty}\left[\psi_{t}\left(L^{*}\right) g\right]\left[\phi_{t}(L) f \phi_{t}(L) h\right] \frac{d t}{t} d \mu,
$$

is bounded on $L^{p} \times L^{q} \times L^{r}$ for all exponents $p, q, r \in(1, \infty)$ satisfying

$$
\frac{1}{p}+\frac{1}{q}+\frac{1}{r}=1
$$

Equivalently, the paraproduct

$$
(h, f) \rightarrow \int_{0}^{\infty} \psi_{t}(L)\left[\phi_{t}(L) f \phi_{t}(L) h\right] \frac{d t}{t}
$$

is bounded from $L^{p} \times L^{q}$ to $L^{r^{\prime}}$.

Moreover $p$ or $q$ may be infinite.

Proof. By trilinear interpolation, it suffices for us to prove boundedness for the limiting case: when one of the exponents is infinite. By symmetry between $f$ and $h$, we also have to deal with only one case: when $p=\infty$ (Step 1) and then conclude by interpolation (Step 2).

Step 1. Estimate for $p=\infty$.

Let us fix $h \in L^{\infty}$ and consider the operator $U$ defined by

$$
U(f):=\int_{0}^{\infty} \psi_{t}(L)\left[\phi_{t}(L) f \phi_{t}(L) h\right] \frac{d t}{t}
$$

in order that

$$
\Lambda(h, f, g):=\langle U(f), g\rangle .
$$

We will prove that $U$ satisfies the assumptions of Theorem 3.3. Then the desired result will follow from Corollary 3.6. First, $L(\mathbf{1})=0$ yields

$$
U(\mathbf{1})=\int_{0}^{\infty} \psi_{t}(L) \phi_{t}(L) h \frac{d t}{t}=c h \in L^{\infty} \subset B M O_{L},
$$

where $c:=\int_{0}^{\infty} \psi_{t}(x) \phi_{t}(x) \frac{d t}{t}$ is a numerical constant independent of $x$. Moreover, it is obvious that

$$
U^{*}(\mathbf{1})=0 .
$$

So it remains to check assumptions (3.1), (3.2) and (3.3), which we recall here.

Consider a large enough integer $\kappa$. For balls $Q_{1}, Q_{2}$ of radius $r=s^{1 / m}$, we have

- if $d\left(Q_{1}, Q_{2}\right) \geq 2 r$, then

$$
\left\|(s L)^{\kappa} e^{-s L} U(f)\right\|_{L^{2}\left(Q_{2}\right)} \lesssim\left(1+\frac{d\left(Q_{1}, Q_{2}\right)}{r}\right)^{-d-2 N-\delta}\|f\|_{L^{2}\left(Q_{1}\right)},
$$

and the dual estimates

$$
\left\|\left(s L^{*}\right)^{\kappa} e^{-s L^{*}} U^{*}(f)\right\|_{L^{2}\left(Q_{2}\right)} \lesssim\left(1+\frac{d\left(Q_{1}, Q_{2}\right)}{r}\right)^{-d-2 N-\delta}\|f\|_{L^{2}\left(Q_{1}\right)} ;
$$

- if $d\left(Q_{1}, Q_{2}\right) \leq 2 r$, then we have

$$
\left\|(s L)^{\kappa} e^{-s L} U\left(e^{-s L} f\right)\right\|_{L^{2}\left(Q_{2}\right)}+\left\|\left(s L^{*}\right)^{\kappa} e^{-s L^{*}} U\left(e^{-s L^{*}} f\right)\right\|_{L^{2}\left(Q_{2}\right)} \lesssim\|f\|_{L^{2}\left(Q_{1}\right)} \text {. }
$$


Step 1-1. Assumption (4.2).

The operator $U$ is given by

$$
U(f):=\int_{0}^{\infty} \psi_{t}(L)\left[\phi_{t}(L) f \phi_{t}(L) h\right] \frac{d t}{t} .
$$

We divide the integral on $t$ as follows:

$$
\left(\int_{Q_{2}}\left|(s L)^{\kappa} e^{-s L} U(f)\right|^{2} d \mu\right)^{1 / 2} \lesssim I+I I
$$

with

$$
I:=\int_{0}^{s}\left\|s^{\kappa} t^{N} L^{\kappa+N} e^{-(s+t) L}\left(1-e^{-t L}\right)\left[\phi_{t}(L) f \phi_{t}(L) h\right]\right\|_{L^{2}\left(Q_{2}\right)} \frac{d t}{t}
$$

and

$$
I I:=\int_{s}^{\infty}\left\|s^{\kappa} t^{N} L^{\kappa+N} e^{-(s+t) L}\left(1-e^{-t L}\right)\left[\phi_{t}(L) f \phi_{t}(L) h\right]\right\|_{L^{2}\left(Q_{2}\right)} \frac{d t}{t} .
$$

Let us treat the first term $I$ (the reasoning is similar to the one used for Corollary 3.6).

Thanks to the $L^{2}$-off-diagonal decay of the semigroup (and its derivative), we have, since $t+s \in[s, 2 s]$,

$$
\begin{aligned}
I & \lesssim \int_{0}^{s}\left\|s^{\kappa} t^{N} L^{\kappa+N} e^{-(s+t) L}\left(1-e^{-t L}\right)\left[\phi_{t}(L) f \phi_{t}(L) h\right]\right\|_{L^{2}\left(Q_{2}\right)} \frac{d t}{t} \\
& \lesssim \int_{0}^{s} \frac{s^{\kappa} t^{N}}{(s+t)^{\kappa+N}}\left\|(s+t)^{\kappa+N} L^{\kappa+N} e^{-(s+t) L}\left(1-e^{-t L}\right)\left[\phi_{t}(L) f \phi_{t}(L) h\right]\right\|_{L^{2}\left(Q_{2}\right)} \frac{d t}{t} .
\end{aligned}
$$

Now let $t \in(0, s]$. Then $s+t \in[s, 2 s]$, so we know that $(s+t)^{\kappa+N} L^{\kappa+N} e^{-(s+t) L}$ satisfies $L^{2}-L^{2}$ off-diagonal decay at the scale $s$. Hence by considering $\left(R_{k}\right)_{k}$ to be a covering of the whole space $M$ by balls of radius $r$, we get

$$
\begin{aligned}
\left\|(s+t)^{\kappa+N} L^{\kappa+N} e^{-(s+t) L}\left(1-e^{-t L}\right)\left[\phi_{t}(L) f \phi_{t}(L) h\right]\right\|_{L^{2}\left(Q_{2}\right)} \\
\quad \lesssim \sum_{k}\left(1+\frac{d\left(Q_{2}, R_{k}\right)}{r}\right)^{-d-2 N-\delta}\left\|\left(1-e^{-t L}\right)\left[\phi_{t}(L) f \phi_{t}(L) h\right]\right\|_{L^{2}\left(R_{k}\right)} .
\end{aligned}
$$

Since $\left(1-e^{-t L}\right)$ and $\phi_{t}(L)$ satisfy $L^{2}-L^{2}$ off-diagonal decays at the scale $t$ and $\phi_{t}(L) h$ is pointwisely bounded, we have that $f \rightarrow\left(1-e^{-t L}\right)\left[\phi_{t}(L) f \phi_{t}(L) h\right]$ satisfies similar $L^{2}-L^{2}$ off-diagonal decays. So let $\left(R_{k}^{j}\right)_{j}$ be a bounded covering of $R_{k}$ by 
balls of radius $t^{1 / m}$ (and similarly for $Q_{1}$ ). This yields

$$
\begin{aligned}
\|\left(1-e^{-t L}\right)\left[\phi_{t}(L) f\right. & \left.\phi_{t}(L) h\right] \|_{L^{2}\left(R_{k}\right)} \\
& \lesssim\left(\sum_{j_{1}}\left\|\left(1-e^{-t L}\right)\left[\phi_{t}(L) f \phi_{t}(L) h\right]\right\|_{L^{2}\left(R_{k}^{j_{1}}\right)}^{2}\right)^{1 / 2} \\
& \lesssim\left(\sum_{j_{1}}\left(\sum_{j_{2}}\left(1+\frac{d\left(R_{k}^{j_{1}}, Q_{1}^{j_{2}}\right)}{t^{1 / m}}\right)^{-d-2 N-\delta}\|f\|_{L^{2}\left(Q_{1}^{j_{2}}\right)}\right)^{2}\right)^{1 / 2} \\
& \lesssim\left(1+\frac{d\left(R_{k}, Q_{1}\right)}{t^{1 / m}}\right)^{-d-2 N-\delta}\left(\sum_{j_{1}}\left(\sum_{j_{2}}\|f\|_{L^{2}\left(Q_{1}^{j_{2}}\right)}\right)^{2}\right)^{1 / 2} \\
& \lesssim\left(1+\frac{d\left(R_{k}, Q_{1}\right)}{t^{1 / m}}\right)^{-d-2 N-\delta}\|f\|_{L^{2}\left(Q_{1}\right)}\left(\sum_{j_{1}, j_{2}} 1\right)^{1 / 2} \\
& \lesssim\left(1+\frac{d\left(R_{k}, Q_{1}\right)}{t^{1 / m}}\right)^{-d-2 N-\delta}\|f\|_{L^{2}\left(Q_{1}\right)}\left(\frac{s}{t}\right)^{d / m}
\end{aligned}
$$

where we refer the reader to (3.8) for the estimate of the sum over $j_{1}, j_{2}$. Finally, we get

$$
\begin{aligned}
& \left\|(s+t)^{\kappa+N} L^{\kappa+N} e^{-(s+t) L}\left(1-e^{-t L}\right)\left[\phi_{t}(L) f \phi_{t}(L) h\right]\right\|_{L^{2}\left(Q_{2}\right)} \\
& \quad \lesssim \sum_{k}\left(1+\frac{d\left(Q_{2}, R_{k}\right)}{s^{1 / m}}\right)^{-d-2 N-\delta}\left(1+\frac{d\left(R_{k}, Q_{1}\right)}{t^{1 / m}}\right)^{-d-2 N-\delta}\|f\|_{L^{2}\left(Q_{1}\right)}\left(\frac{s}{t}\right)^{d / m} \\
& \quad \lesssim\left(1+\frac{d\left(Q_{2}, Q_{1}\right)}{r}\right)^{-d-2 N-\delta}\left(\frac{s}{t}\right)^{d / m}\|f\|_{L^{2}\left(Q_{1}\right)} .
\end{aligned}
$$

This permits us to deduce (4.2) for the first term $I$ since $N>d / m$ and

$$
\int_{0}^{s} \frac{s^{\kappa} t^{N}}{(s+t)^{\kappa+N}}\left(\frac{s}{t}\right)^{d / m} \frac{d t}{t} \lesssim 1
$$

Concerning the second term $I I$, we produce a similar reasoning and we deduce that for $t \geq s$,

$$
\begin{aligned}
\|(s+t)^{\kappa+N} L^{\kappa+N} e^{-(s+t) L}\left(1-e^{-t L}\right) & {\left[\phi_{t}(L) f \phi_{t}(L) h\right] \|_{L^{2}\left(Q_{2}\right)} } \\
& \lesssim\left(1+\frac{d\left(Q_{2}, Q_{1}\right)}{t^{1 / m}}\right)^{-d-2 N-\delta}\|f\|_{L^{2}\left(Q_{1}\right)} .
\end{aligned}
$$

Indeed, each appearing operator admits off-diagonal decay at the scale $t^{1 / m}$ since $s+t \simeq t$. We also prove (4.2) for the first term $I I$ since for $\kappa \geq d+2 N+\delta$,

$$
\int_{s}^{\infty} \frac{s^{\kappa} t^{N}}{(s+t)^{\kappa+N}}\left(1+\frac{d\left(Q_{2}, Q_{1}\right)}{t^{1 / m}}\right)^{-d-2 N-\delta} \frac{d t}{t} \lesssim\left(1+\frac{d\left(Q_{2}, Q_{1}\right)}{r}\right)^{-d-2 N-\delta} .
$$

We have also finished checking assumption (4.2). 
Step 1-2. Assumption (4.3).

By duality, (4.3) is equivalent to

$$
\left\|U\left((s L)^{\kappa} e^{-s L} f\right)\right\|_{L^{2}\left(Q_{2}\right)} \lesssim\left(1+\frac{d\left(Q_{1}, Q_{2}\right)}{r}\right)^{-d-2 N-\delta}\|f\|_{L^{2}\left(Q_{1}\right)},
$$

which we are going to prove.

We will just give the sketch of the proof since the technical details are by now routine. As previously, the quantity to estimate can be divided into two quantities $I^{*}$ and $I I^{*}$ with

$$
I^{*}:=\left\|\int_{0}^{s}(t L)^{N} e^{-t L}\left(1-e^{-t L}\right)\left[(s L)^{\kappa} e^{-s L} \phi_{t}(L) f \phi_{t}(L) h\right] \frac{d t}{t}\right\|_{L^{2}\left(Q_{2}\right)}
$$

and

$$
I I^{*}:=\int_{s}^{\infty}\left\|(t L)^{N} e^{-t L}\left(1-e^{-t L}\right)\left[(s L)^{\kappa} e^{-s L} \phi_{t}(L) f \phi_{t}(L) h\right]\right\|_{L^{2}\left(Q_{2}\right)} \frac{d t}{t} .
$$

The second quantity can be exactly estimated as $I I$ in the previous point and so we only deal with the first quantity. First, it is easy to check that we can replace the above quantity

$$
(t L)^{N} e^{-t L}\left(1-e^{-t L}\right)\left[(s L)^{\kappa} e^{-s L} \phi_{t}(L) f \phi_{t}(L) h\right]
$$

by

$$
(t L)^{N} e^{-t L}\left(1-e^{-t L}\right)\left[\tilde{\psi}_{s}(L) f \phi_{t}(L) h\right]
$$

where $\tilde{\psi}_{s}(L)=(s L)^{\kappa} e^{-s L}$ since $(s L)^{\kappa} e^{-s L} \phi_{t}(L)=(s L)^{\kappa} e^{-(s+t) L}$ and $t \in(0, s]$. Indeed by computing the difference, it appears that

$$
e^{-s L}-e^{-(s+t) L} \simeq t L e^{-s L}=\frac{t}{s}(s L) e^{-s L},
$$

involving an extra factor $\frac{t}{s}$ which permits us to easily bound the difference as desired. So let us just consider

$$
\left\|\int_{0}^{s}(t L)^{N} e^{-t L}\left(1-e^{-t L}\right)\left[\tilde{\psi}_{s}(L) f \phi_{t}(L) h\right] \frac{d t}{t}\right\|_{L^{2}\left(Q_{2}\right)} .
$$

Since $\tilde{\psi}_{s}(L) f$ is essentially constant at the scale $t \ll s$, we can compare the previous quantity to the following one:

$$
\left\|\tilde{\psi}_{s}(L) f \int_{0}^{s}(t L)^{N} e^{-t L}\left(1-e^{-t L}\right)\left[\phi_{t}(L) h\right] \frac{d t}{t}\right\|_{L^{2}\left(Q_{2}\right)},
$$

which satisfies the desired estimate since $\int_{0}^{s}(t L)^{N} e^{-t L}\left(1-e^{-t L}\right)\left[\phi_{t}(L) h\right] \frac{d t}{t}$ is a uniformly bounded function for $h \in L^{\infty}$. It also remains for us to study the difference between (4.6) and (4.7). We leave the details to the reader, but the analysis of the difference is based on exactly the same arguments as used for the study of $I_{s}$, in the proof of Theorem 3.3 . The difference makes the gradient $\nabla \tilde{\psi}_{s}(L) f$ appear at the scale $t$, so we obtain an extra decay such as

$$
t^{1 / m} \nabla \tilde{\psi}_{s}(L) f=\left(\frac{t}{s}\right)^{1 / m} s^{1 / m} \nabla \tilde{\psi}_{s}(L) f .
$$

By assumption (4.1), we obtain the desired off-diagonal decays, and the extra factor $\left(\frac{t}{s}\right)^{1 / m}$ permits us to make the integral on $t$ convergent one more time. 
Step 1-3. Assumption (4.4).

We leave it to the reader to check that the two previous points (Steps 1-1 and 1-2) still hold when $d\left(Q_{1}, Q_{2}\right) \leq r$ and permit us to prove (4.4).

This finishes the proof of Step 1 and, by Corollary [3.6, it yields the desired estimates for $p=\infty$.

Step 2. End of the proof.

By symmetry between $f$ and $h$, we know that the trilinear form $\Lambda$ is bounded on $L^{\infty} \times L^{q} \times L^{q^{\prime}}$ (Step 1) and on $L^{p} \times L^{\infty} \times L^{p^{\prime}}$ (by symmetry). So for $r \in(1, \infty)$ fixed, we know that $\Lambda$ is bounded on $L^{\infty} \times L^{r^{\prime}} \times L^{r}$ and on $L^{r^{\prime}} \times L^{\infty} \times L^{r}$, which by bilinear interpolation gives a boundedness on $L^{p} \times L^{q} \times L^{r}$.

By duality, we have the following results:

Theorem 4.6. The trilinear form $\Lambda$, defined by

$$
\Lambda(h, f, g):=\int_{0}^{\infty} \int_{M}\left[\phi_{t}\left(L^{*}\right) g\right]\left[\psi_{t}(L) f \phi_{t}(L) h\right] \frac{d \mu d t}{t},
$$

is bounded on $L^{p} \times L^{q} \times L^{r}$ for all exponents $p, q, r \in(1, \infty)$ satisfying

$$
\frac{1}{p}+\frac{1}{q}+\frac{1}{r}=1
$$

Equivalently, the paraproduct

$$
(h, f) \rightarrow \int_{0}^{\infty} \phi_{t}(L)\left[\psi_{t}(L) f \phi_{t}(L) h\right] \frac{d t}{t}
$$

is bounded from $L^{p} \times L^{q}$ to $L^{r^{\prime}}$.

Moreover $p$ or $q$ may be infinite.

Remark 4.7. The trilinear forms of Theorems 4.5 and 4.6 appear naturally in the study of the product. For example, let $\phi$ be the function

$$
\phi(x):=-\int_{x}^{\infty} y e^{-y}\left(1-e^{-y}\right) d y .
$$

We leave it to the reader to check that all the previous results still hold with the new operator

$$
\phi_{t}(L):=\phi(t L)
$$

(instead of $\left.\phi_{t}(L)=e^{-t L}\right)$. Then we get a "spectral" decomposition of the identity as follows: up to some numerical constant $c$, we have

$$
f=c \int_{0}^{\infty} \phi^{\prime}(t L) f \frac{d t}{t}
$$

according to Remark 2.8. So for two smooth functions, we have

$$
f g:=c^{3} \int_{s, u, v>0} \phi^{\prime}(s L)\left[\phi^{\prime}(u L) f \phi^{\prime}(v L) g\right] \frac{d s d u d v}{s u v} .
$$

Since $\phi^{\prime}(x)=\psi(x):=x e^{-x}\left(1-e^{-x}\right)$, it follows that (by integrating according to $t:=\min \{s, u, v\})$

$$
\begin{aligned}
f g: & =c^{3} \int_{0}^{\infty} \psi(t L)[\phi(t L) f \phi(t L) g] \frac{d t}{t}+c^{3} \int_{0}^{\infty} \phi(t L)[\psi(t L) f \phi(t L) g] \frac{d t}{t} \\
& +c^{3} \int_{0}^{\infty} \phi(t L)[\phi(t L) f \psi(t L) g] \frac{d t}{t} .
\end{aligned}
$$


Consequently, the pointwise product $f g$ can be decomposed with tree paraproducts (involving only one function $\psi$ ), studied by Theorems 4.5 and 4.6 .

4.2. Boundedness in weighted Lebesgue spaces and extrapolation to the case $r^{\prime} \leq 1$. We are now interested in extending previous results to weighted Lebesgue spaces and with exponents $r^{\prime} \leq 1$. We refer the reader to Definition 3.7 for the usual class of weights.

Proposition 4.8. Let $\psi$ and $\phi$ be defined as in Theorems 4.5 and 4.6. Let $p, q, r \in$ $(1, \infty)$ be exponents satisfying

$$
\frac{1}{p}+\frac{1}{q}+\frac{1}{r}=1
$$

and consider a weight $\omega$ belonging to $\mathbb{A}_{p} \cap \mathbb{A}_{q}$. The paraproducts

$$
(h, f) \rightarrow \int_{0}^{\infty} \psi_{t}(L)\left[\phi_{t}(L) f \phi_{t}(L) h\right] \frac{d t}{t}
$$

and

$$
(h, f) \rightarrow \int_{0}^{\infty} \phi_{t}(L)\left[\psi_{t}(L) f \phi_{t}(L) h\right] \frac{d t}{t}
$$

are bounded from $L^{p}(\omega) \times L^{q}(\omega)$ to $L^{r^{\prime}}(\omega)$.

Proof. Theorems 4.5 and 4.6 correspond to the desired result with the constant weight $\omega=1$.

It is well known that weighted estimates are closely related to estimates of some maximal sharp functions. Let us denote the following maximal sharp function associated to an exponent $s \in[1, \infty)$ (introduced by J.M. Martell in [44] and extended in [10]):

$$
M_{s}^{\sharp}(h)(x):=\left(\sup _{t>0} \frac{1}{B\left(x, t^{1 / m}\right)} \int_{B\left(x, t^{1 / m}\right)}|\psi(t L)(h)|^{s} d \mu\right)^{1 / s} .
$$

Let us explain how we can obtain the desired result only for the first paraproduct (the reasoning for the second one being similar):

$$
T(h, f):=\int_{0}^{\infty} \psi_{t}(L)\left[\phi_{t}(L) f \phi_{t}(L) h\right] \frac{d t}{t} .
$$

The $L^{2}-L^{2}$ off-diagonal decays (4.2), (4.3) and (4.4) yield that

$$
M_{2}^{\sharp}(T(h, f))(x) \lesssim \mathcal{M}_{2}(f)(x) \mathcal{M}_{2}(h)(x) .
$$

We leave it to the reader to check this point, but it is a direct consequence of the off-diagonal decays and the pointwise bound of the heat kernel (see Theorem 6.1 in [10] for a detailed proof of such inequalities). In addition, since we assume $L^{1}-L^{\infty}$ off-diagonal decays of the semigroup and its derivatives (pointwise estimates of the heat kernel), it is easy to see that we can obtain off-diagonal decays (4.2), (4.3) and (4.4) for all exponents $s \in(1, \infty)$ and not only for $s=2$. So we can obtain an estimate such as

$$
M_{s}^{\sharp}(T(h, f))(x) \lesssim \mathcal{M}_{s}(f)(x) \mathcal{M}_{s}(h)(x)
$$

for every exponent $s>1$. 
In order to compare the Lebesgue norm of $T(h, f)$ and the one of $M_{s}^{\sharp}(T(h, f))$, we need to use a Fefferman-Stein inequality. We refer the reader to 44] (Theorem 4.2), [10] (Corollary 5.8 and Theorem 6.4) and to [11] (Lemma 2, Remark 3 and Lemma 3 for the weighted version) for such inequalities. From pointwise estimates on the semigroup and Step 3 in the proof of Corollary 3.6, we know that for every $s \in(1, \infty)$ and weight $\nu$,

$$
\left\|\mathcal{M}_{s}[T(h, f)]\right\|_{L^{r}(\nu)} \lesssim\left\|M_{s}^{\sharp}[T(h, f)]\right\|_{L^{r}(\nu)} .
$$

Such inequalities are based on "good- $\lambda$ inequalities" relative to the two maximal operators, obtained in a very general framework by P. Auscher and J.M. Martell in [7] (Theorem 3.1).

Consequently, for our weight $\omega$, the Hölder inequality and (4.8) give

$$
\begin{aligned}
\|T(h, f)\|_{L^{r}(\omega)} & \leq\left\|\mathcal{M}_{s}[T(h, f)]\right\|_{L^{r}(\omega)} \\
& \lesssim\left\|M_{s}^{\sharp}[T(h, f)]\right\|_{L^{r}(\omega)} \\
& \lesssim\left\|\mathcal{M}_{s}(f) \mathcal{M}_{s}(h)\right\|_{L^{r}(\omega)} \\
& \lesssim\left\|\mathcal{M}_{s}(f)\right\|_{L^{q}(\omega)}\left\|\mathcal{M}_{s}(h)\right\|_{L^{p}(\omega)} .
\end{aligned}
$$

Then we choose $s \in(1, \min \{p, q\})$ in order that $\mathcal{M}_{s}$ is bounded in $L^{p}(\omega)$ and in $L^{q}(\omega)$ (due to $\omega \in \mathbb{A}_{p} \cap \mathbb{A}_{q}$ ) and so we conclude that $T$ is bounded from $L^{p}(\omega) \times L^{q}(\omega)$ into $L^{r^{\prime}}(\omega)$.

Remark 4.9. Using recent works of L. Grafakos, L. Liu, A. Lerner, S. Ombrosi, C. Pérez, R. H. Torres and R. Trujillo-González [41, 31, it seems possible to get similar results with different weights for $h$ and $f$. This requires the notion of a bilinear $\mathbb{A}_{\vec{P}}$ condition and the use of a "bilinear strong maximal function". We do not detail these possible improvements here.

Then we use the theory of extrapolation to obtain new boundedness for our paraproducts (see Theorem 2 of [30]):

Theorem 4.10. Let $\psi$ and $\phi$ be defined as in Theorems 4.5 and 4.6. Let $p, q \in$ $(1, \infty)$ and $r^{\prime} \in(1 / 2, \infty)$ be exponents satisfying

$$
\frac{1}{p}+\frac{1}{q}=\frac{1}{r^{\prime}}
$$

and consider a weight $\omega$ belonging to $\mathbb{A}_{p} \cap \mathbb{A}_{q}$. The paraproducts

$$
(h, f) \rightarrow \int_{0}^{\infty} \psi_{t}(L)\left[\phi_{t}(L) f \phi_{t}(L) h\right] \frac{d t}{t}
$$

and

$$
(h, f) \rightarrow \int_{0}^{\infty} \phi_{t}(L)\left[\psi_{t}(L) f \phi_{t}(L) h\right] \frac{d t}{t}
$$

are bounded from $L^{p}(\omega) \times L^{q}(\omega)$ to $L^{r^{\prime}}(\omega)$.

Remark 4.11. The improvement in this new result is that the exponent $r^{\prime}$ could be smaller than one. 
4.3. A "classical" $T(1)$-theorem on a Riemannian manifold. We devote this subsection to the proof of a $T(1)$-theorem for Calderón-Zygmund operators on a general doubling Riemannian manifold $(M, d, \mu)$ of infinite measure.

Definition 4.12. A function $K$ defined on $M \times M \backslash\{(x, x): x \in M\}$ is called a "standard kernel of order $1+\epsilon$ " for some $\epsilon \in(0,1]$ if for all $x \neq y$,

$$
|K(x, y)| \lesssim \frac{1}{d(x, y)^{d+3 N}}
$$

for $x^{\prime} \in M$ satisfying $\left|x-x^{\prime}\right| \leq \frac{1}{2} \max \left\{|x-y|,\left|x^{\prime}-y\right|\right\}$,

$$
\left|\nabla K(x, y)-\nabla K\left(x^{\prime}, y\right)\right| \lesssim \frac{d\left(x, x^{\prime}\right)^{\epsilon}}{\left(d(x, y)+d\left(x^{\prime}, y\right)\right)^{d+3 N+1+\epsilon}}
$$

and for $y^{\prime} \in M$ satisfying $\left|y-y^{\prime}\right| \leq \frac{1}{2} \max \left\{|x-y|,\left|x-y^{\prime}\right|\right\}$,

$$
\left|\nabla K(x, y)-\nabla K\left(x, y^{\prime}\right)\right| \lesssim \frac{d\left(y, y^{\prime}\right)^{\epsilon}}{\left(d(x, y)+d\left(x, y^{\prime}\right)\right)^{d+3 N+1+\epsilon}} .
$$

A linear operator $T$, continuously acting from $\mathcal{M}$ to $\mathcal{S}^{\prime}(M)$ and satisfying the integral representation

$$
\forall f \in C_{0}^{\infty}(M), \forall x \notin \operatorname{supp}(f) \quad T(f)(x)=\int_{M} K(x, y) f(y) d \mu(y),
$$

is said to be associated to the kernel $K$.

Theorem 4.13. Let us assume that the doubling manifold $M$ satisfies Poincaré $\left(P_{2}\right)$ and assumption (2.8), on its heat kernel. For $T$ a linear operator associated to a standard kernel of order $1+\epsilon$ (such that $T(\mathbf{1})$ and $T^{*}(\mathbf{1})$ are well defined in $\mathbb{M})$, the following two properties are equivalent:

- $T$ is bounded on $L^{2}$;

- $T(\mathbf{1})$ and $T^{*}(\mathbf{1})$ belong to $B M O_{-\Delta}$ and $T$ satisfies the weak-boundedness property (3.3).

Since $B M O \subset B M O_{-\Delta}$, if $T(\mathbf{1})$ and $T^{*}(\mathbf{1})$ belong to $B M O$, then they belong to $\mathrm{BMO}_{-\Delta}$ too.

Proof. We look to apply our new $T(1)$-theorem as follows. Let us consider $L=-\Delta$ given by the Laplacian. Then we have seen in Subsubsection 2.5.2 that under (2.8) all our required assumptions are satisfied by the heat semigroup $\left(e^{-t L}\right)_{t>0}$ with $m=2$. Moreover, we know that we have a pointwise Gaussian bound of the heat kernel $p_{t}$ :

$$
\left|p_{t}(x, y)\right| \lesssim e^{-\gamma d(x, y)^{2} / t}
$$

for some constant $\gamma>0$.

Since $B M O$ is included in $B M O_{L}$ (see Proposition 6.7 in 23 and Remark 7.6 of [10), it remains for us to check that our operator $T$ satisfies (3.1), (3.2) with $\kappa=1$. By duality and symmetry, we only deal with (3.2): for every $s>0$ and all balls $Q_{1}, Q_{2}$ of radius $r:=s^{1 / 2}$ (with $d\left(Q_{1}, Q_{2}\right) \geq 2 r$ ) and functions $f \in L^{2}\left(Q_{1}\right)$,

$$
\left\|(-s \Delta) e^{s \Delta} T(f)\right\|_{L^{2}\left(Q_{2}\right)} \lesssim\left(1+\frac{d\left(Q_{1}, Q_{2}\right)}{r}\right)^{-d-2 N-\delta}\|f\|_{L^{2}\left(Q_{1}\right)} .
$$


So let us consider the balls $Q_{1}$ and $Q_{2}$ and write for $x_{0} \in Q_{2}$,

$$
\begin{aligned}
(-s \Delta) e^{s \Delta} T(f)\left(x_{0}\right) & =-\int s \Delta_{x} p_{s}\left(x_{0}, y\right) T(f)(y) d \mu(y) \\
& =-\int s \Delta_{y} p_{s}\left(x_{0}, y\right) T(f)(y) d \mu(y) \\
& =\int s \nabla_{y} p_{s}\left(x_{0}, y\right) \nabla T(f)(y) d \mu(y) \\
& =\int s \nabla_{y} p_{s}\left(x_{0}, y\right)\left[\nabla T(f)(y)-\nabla T(f)\left(x_{0}\right)\right] d \mu(y),
\end{aligned}
$$

where we used the self-adjoint properties of the Laplacian, an integration by parts and at the last line the fact that

$$
\int \nabla_{y} p_{s}\left(x_{0}, y\right) d \mu(y)=0
$$

So we have

$$
\begin{aligned}
& \left|(-s \Delta) e^{s \Delta} T(f)\left(x_{0}\right)\right| \\
& \quad \leq \iint s\left|\nabla_{y} p_{s}\left(x_{0}, y\right)\right|\left|\nabla_{y} K(y, z)-\nabla_{y} K\left(x_{0}, z\right)\right||f(z)| d \mu(y) d \mu(z) .
\end{aligned}
$$

Using the properties of the standard kernel, we deduce that

$$
\begin{aligned}
& \left|(-s \Delta) e^{s \Delta} T(f)\left(x_{0}\right)\right| \\
& \quad \leq \iint s\left|\nabla_{y} p_{s}\left(x_{0}, y\right)\right| \frac{d\left(y, x_{0}\right)^{\epsilon}}{\left(d(y, z)+d\left(x_{0}, z\right)\right)^{d+3 N+1+\epsilon}}|f(z)| d \mu(y) d \mu(z) .
\end{aligned}
$$

Then using the Cauchy-Schwarz inequality and the weighted estimates on the gradient of the heat kernel (see Lemma 2.2 of [19]), we have

$$
\int\left|\nabla_{y} p_{s}\left(x_{0}, y\right)\right|^{2} e^{\gamma d\left(x_{0}, y\right)^{2} /(2 s)} d \mu(y) \lesssim \mu\left(B\left(x_{0}, r\right)\right)^{-1 / 2} .
$$

We obtain $\left(\right.$ since $\left.d\left(x_{0}, z\right) \geq d\left(Q_{2}, Q_{1}\right) \geq 2 r\right)$ :

$$
\left|(-s \Delta) e^{s \Delta} T(f)\left(x_{0}\right)\right| \lesssim \frac{1}{\mu\left(B\left(x_{0}, r\right)\right)} \int \frac{1}{\left(1+\frac{d\left(x_{0}, z\right)}{r}\right)^{d+3 N+1+\epsilon}}|f(z)| d \mu(z),
$$

which yields (4.9) by integrating over $x_{0} \in Q_{2}$.

Usually, for a linear operator $T$ associated to a standard kernel $K$, we use the following weak boundedness property: for all smooth functions $f, g$ such that for some $x_{0} \in M$ and $R>0$ and every $\alpha$,

$$
\left|\nabla^{\alpha} f(x)\right| \lesssim \mu\left(B\left(x_{0}, R\right)\right)^{-d-|\alpha|}\left(1+\frac{d\left(x, x_{0}\right)}{R}\right)^{-N}
$$

for a large enough integer $N$ (and similarly for $g$ ), we have

$$
|\langle T(f), g\rangle| \lesssim \frac{1}{\mu\left(B\left(x_{0}, R\right)\right)}
$$

Indeed this property implies our (3.3), since $e^{-t L}(f)$ is a smooth function at the scale $t^{1 / 2}$. We also recover the classical $T(1)$-theorem in Euclidean space and extend it on a large class of Riemannian manifolds. It could be interesting to make a mixture of our present study with the works of F. Nazarov, S. Treil and A. Volberg 
47, 48] and X. Tolsa [52] in order to obtain results in Riemannian manifolds with a non-doubling measure.

We finish this work by asking an open question: in some situations, the semigroup $\left(e^{-t L}\right)_{t>0}$ does not satisfy pointwise estimates such as (2.4) but only $L^{2}-L^{2}$ off-diagonal estimates (such as Gaffney estimates). Can we expect a similar T(1)theorem under just off-diagonal decays for the heat kernel? In our proof, the pointwise bound seems to be very important on the one hand to get a Sobolev inequality (Proposition 3.10) and on the other hand to bound the maximal function (3.16) appearing in the "Carleson measure - argument".

\section{REFERENCES}

1. L. Ambrosio, M. Miranda, Jr. and D. Pallara, Special functions of bounded variation in doubling metric measure spaces, Calculus of variations: topics from the mathematical heritage of E. De Giorgi, Quad. Mat., Dept. Math., Seconda Univ. Napoli, Caserta 14 (2004), 1-45. MR2118414 (2005j:49036)

2. P. Auscher and P. Tchamitchian, Square root problem for divergence operators and related topics, Astérisque 249 (1998), Soc. Math. France. MR1651262 (2000c:47092)

3. P. Auscher, On $L^{p}$ estimates for square roots of second order elliptic operators on $\mathbb{R}^{n}$, Publ. Mat. 48 (2004), 159-186. MR2044643 (2005m:35065)

4. P. Auscher, On necessary and sufficient conditions for $L^{p}$ estimates of Riesz transforms associated to elliptic operators on $\mathbb{R}^{n}$ and related estimates, Memoirs of Amer. Math. Soc. 186 no. 871 (2007). MR $2292385(2007 \mathrm{k}: 42025)$

5. P. Auscher, T. Coulhon, X.T. Duong, and S. Hofmann, Riesz transform on manifolds and the heat kernel regularity, Ann. Sci. Ecole Norm. Sup. 37 (2004), 911-957. MR 2119242 (2005k:58043)

6. P. Auscher, A. McIntosh and E. Russ, Hardy spaces of differential forms on Riemannian manifolds, Journ. of Geom. Anal. 18 (2008), 192-248. MR2365673 (2009d:42053)

7. P. Auscher and J.M. Martell, Weighted norm inequalities, off-diagonal estimates and elliptic operators, Part I : General operator theory and weights, Adv. in Math. 212 (2007), 225-276. MR2319768 (2008m:42015)

8. P. Auscher and E. Russ, Hardy spaces and divergence operators on strongly Lipschitz domains of $\mathbb{R}^{n}$, J. Funct. Anal. 201 (2003), no. 1, 148-184. MR1986158(2004c:42049)

9. N. Badr and F. Bernicot, Abstract Hardy-Sobolev spaces and interpolation, Journ. Funct. Anal. 259 (2010), no. 5, 1169-1208. MR2652185

10. F. Bernicot and J. Zhao, New Abstract Hardy Spaces, J. Funct. Anal. 255 (2008), 1761-1796. MR 2442082(2009k:46043)

11. F. Bernicot, Use of abstract Hardy spaces, real interpolation and applications to bilinear operators, Math. Z. 265 (2010), 365-400. MR2609316 (2011c:42058)

12. F. Bernicot and J. Zhao, Abstract framework for John Nirenberg inequalities and applications to Hardy spaces, Ann. Sc. Norm. Super. Pisa Cl. Sci. (2012) available at http://arxiv.org/abs/1003.0591.

13. J.-M. Bony, Calcul symbolique et propagation des singularités pour les équations aux dérivées partielles non linéaires, Ann. Sci. Ecole Norm. Sup. 14 (1981), 209-246. MR631751 (84h:35177)

14. M. Christ, A $T(b)$ theorem with remarks on analytic capacity and the Cauchy integral, Colloquium Mathematicum LX/LXI (1990), 601-628. MR.1096400(92k:42020)

15. R.R. Coifman and Y. Meyer, Au delà des opérateurs pseudo-différentiels, Astérisque 57, Société Math. de France, 1978. MR.518170 (81b:47061)

16. R. Coifman and G. Weiss, Analyse harmonique non-commutative sur certains espaces homogènes, Lecture Notes in Math. 242 (1971). MR0499948 (58:17690)

17. R. Coifman and G. Weiss, Extensions of Hardy spaces and their use in analysis, Bull. Amer. Math. Soc. 83 (1977), 569-645. MR0447954(56:6264)

18. T. Coulhon, Itération de Moser et estimation gaussienne du noyau de la chaleur, Journ. Oper. Th. 29 (1993), 157-165. MR.1277971 (95d:47050)

19. T. Coulhon and X.T. Duong, Riesz transforms for $1 \leq p \leq 2$, Trans. Amer. Math. Soc. 351 (1999), no. 2, 1151-1169. MR.1458299 (99e:58174) 
20. G. David and J.L. Journé, A boundedness criterion for generalized Calderón-Zygmund operators, Ann. of Math. (2) 120 (1984), 371-397. MR763911 (85k:42041)

21. G. David, J.L. Journé and S. Semmes, Opérateurs de Calderón-Zygmund, fonctions paraaccrétives et interpolation Rev. Mat. Iberoamericana 1 (1985), 1-56. MR850408 (88f:47024)

22. E.B. Davies, Non-Gaussian aspects of heat kernel behaviour, J. London Math. Soc. 55 (1997), 105-125. MR1423289 (97i:58169)

23. X.T. Duong and L. Yan, Duality of Hardy and $B M O$ spaces associated with operators with heat kernel bounds, J. Amer. Math. Soc. 18, no.4 (2005), 943-973. MR2163867|(2006d:42037)

24. X.T. Duong and L. Yan, New function spaces of $B M O$ type, the John-Nirenberg inequality, Interpolation and Applications, Comm. on Pures and Appl. Math. 58, no.10 (2005), 13751420. MR2162784 (2006i:26012)

25. J. Dziubański, Atomic decomposition of $H^{p}$ spaces associated with some Schrödinger operators, Indiana Univ. Math. J. 47 (1998), 75-98. MR1631616 (99j:46030)

26. J. Dziubański, Spectral multipliers for Hardy spaces associated with Schrödinger operators with polynomial potentials, Bull. London Math. Soc. 32 (2000), 571-581. MR 1767710 (2001g:47087)

27. J. Dziubański, Note on $H^{1}$ spaces related to degenerate Schrödinger operators, Ill. J. Math. 49, no.4, (2005). MR2210363 (2006m:42037)

28. J. Dziubański and J. Zienkiewicz, Hardy spaces $H^{1}$ for Schrödinger operators with compactly supported potentials, Annali di Math. 184 (2005), 315-326. MR2164260 (2006d:42041)

29. C. Fefferman and E.M. Stein, $H^{p}$ spaces of several variables, Acta Math. 129 (1971), 137-193. MR 0447953(56:6263)

30. L. Grafakos and J.M. Martell, Extrapolation of operators of many variables and applications, J. of Geom. Anal. 14 (2004), no. 1, 19-46. MR2030573 (2004m:42019)

31. L. Grafakos, L. Liu, C. Pérez and R. H. Torres, The multilinear strong maximal function, J. Geom. Anal. 21 (2011), 118-149. MR2755679

32. A. Grigor'yan, Estimates of heat kernels on Riemannian manifolds, in Spectral theory and geometry, London Math. Soc. Lecture Note Series, B. Davies, Y. Safarov, eds., 273 (1999), 140-225. MR.1736868 (2001b:58040)

33. P. Hajlasz and P. Koskela, Sobolev met Poincaré, Mem. Amer. Math. Soc. 145 (2000), no. 688, 1-101. MR:1683160 (2000j:46063)

34. S. Hofmann, An Off-Diagonal T1 theorem and applications, Journ. of Funct. Anal. 160 (1998), no. 2, 581-622. MR1665299 (2000a:42027)

35. S. Hofmann, Local $T b$ theorems and applications in PDE, Proceedings of the International Congress of Mathematicians: Madrid, August 22-30,2006 2 (2006), 1375-1392. MR2275650 (2007k:42030)

36. S. Hofmann and S. Mayboroda, Hardy and $B M O$ spaces associated to divergence form elliptic operators, Math. Ann. 344 (2009), no. 1, 37-116. MR2481054(2009m:42038)

37. T. Hytönen, An operator-valued Tb theorem, Journ. Funct. Anal. 234 (2006), no. 2, 420-463. MR 2216905 (2006k:42024)

38. T. Hytönen and L. Weis, A $T 1$ theorem for integral transformations with operator-valued kernel, Journ. Reine und Angewandte Math. 599 (2006), 155-200. MR2279101|(2007i:42020)

39. F. John and L. Nirenberg, On functions of bounded mean oscillation, Comm. Pure Appl. Math. 14 (1961), 785-799. MR0131498 (24:A1348)

40. S. Keith and X. Zhong, The Poincaré inequality is an open ended condition, Ann. of Math. (2) 167 (2008), no. 2, 575-599. MR2415381(2009e:46028)

41. A. Lerner, S. Ombrosi, C. Pérez, R. H. Torres and R. Trujillo-González, New maximal functions and multiple weights for the multilinear Calderón-Zygmund theory, Adv. in Math. 220 (2009), 1222-1264. MR2483720 (2010f:42024)

42. A. McIntosh, Operators which have an $H_{\infty}$-calculus, Miniconference on operator theory and partial differential equations (1986) Proc. Centre Math. Analysis, Australian National University, Canberra 14, 210-231. MR0912940 (88k:47019)

43. A. McIntosh and Y. Meyer, Algèbres d'opérateurs définis par des intégrales singulières, C.R. Acad. Sci. Paris Sér. I. Math. 301 (1985), 395-397. MR808636 (87b:47053)

44. J.M. Martell, Sharp maximal functions associated with approximations of the identity in spaces of homogeneous type and applications, Studia Math. 161 (2004), 113-145. MR.2033231 (2005b:42016) 
45. Y. Meyer, Régularité des solutions des équations aux dérivées partielles non linéaires, Sém. Bourbaki 22 no. 550, (1979).

46. Y. Meyer, Remarques sur un théorème de J.-M. Bony, Rend. Circ. Mat. Palermo, II. Suppl. 1 (1981), 1-20. MR639462 (83b:35169)

47. F. Nazarov, S. Treil and A. Volberg, The Tb-theorem on non-homogeneous spaces, Acta Math. 190 (2003), no. 2, 151-239. MR1998349 (2005d:30053)

48. F. Nazarov, S. Treil and A. Volberg, Accretive system Tb-theorems on nonhomogeneous spaces, Duke Math. J. 113 (2002), no. 2, 259-312. MR1909219 (2003g:42030)

49. L. Saloff-Coste, Aspects of Sobolev type inequalities, Cambridge Univ., 2001. MR1872526 (2003c:46048)

50. A. Sikora, Sharp pointwise estimates for heat kernels, Quart. J. Math. Oxford 47 (1996), 371-382. MR1412562 (97m:58189)

51. E.M. Stein, Harmonic analysis: Real variable Methods, Orthogonality, and Oscillatory Integrals, Princeton Univ. Press, 1993. MR1232192 (95c:42002)

52. X. Tolsa, Littlewood-Paley Theory and the T(1) Theorem with Non-doubling Measures, Adv. in Math. 164 (2001) no. 1, 57-116. MR1870513(2003e:42029)

53. A. V. Vähäkangas, A $T 1$ theorem for weakly singular integral operators, submitted, available at http://arxiv.org/abs/1001.5072.

54. D. Yang, $T 1$ Theorems on Besov and Triebel-Lizorkin Spaces on Spaces of Homogeneous Type and Their Applications, Z. Anal. Anwendungen 22 (2003), no. 1, 53-72. MR1962076 (2004a:43007)

CNRS - Laboratoire Paul Painlevé, Université Lille 1, 59655 Villeneuve d’AscQ Cedex, France

E-mail address: frederic.bernicot@math.univ-lille1.fr

Current address: CNRS - Laboratoire Jean Leray, Université de Nantes, 2, rue de la Houssinière, 44322 Nantes, cedex 3, France 